\title{
Chitosan/PEO nanofibers electrospun on metallized track-etched membranes: fabrication and characterization
}

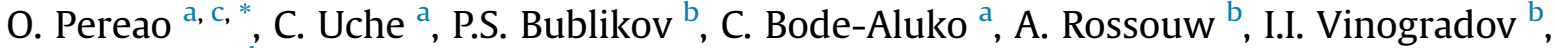 \\ A.N. Nechaev ${ }^{\text {b }}$, B. Opeolu ${ }^{\text {c }}$, L. Petrik ${ }^{\text {a }}$ \\ ${ }^{a}$ Environmental and Nanoscience Research Group, University of the Western Cape, P.B. X17, Bellville, 7535, South Africa \\ ${ }^{\mathrm{b}}$ Flerov Laboratory of Nuclear Reactions, Joint Institute for Nuclear Research, Dubna, 141980, Russia \\ ${ }^{\mathrm{c}}$ Environmental Chemistry and Toxicology Research Group, Cape Peninsula University of Technology, Bellville, 7535, South Africa
}

\section{A R T I C L E I N F O}

\section{Article history:}

Received 10 October 2020

Received in revised form

18 December 2020

Accepted 19 December 2020

Available online $\mathrm{xxx}$

\section{Keywords:}

Electrospinning

Polymer

Water treatment

Polyethylene oxide

Biocompatibility

Daphnia magna

\begin{abstract}
A B S T R A C T
The development of next-generation adsorption, separation, and filtration materials is growing with an increased research focus on polymer composites. In this study, a novel blend of chitosan (CS) and polyethylene oxide (PEO) nanofiber mats was electrospun on titanium (Ti)-coated polyethylene terephthalate (PET) track-etched membranes (TMs) with after-treatment by glutaraldehyde in the vapor phase for enhancing the nanofiber stability by crosslinking. The prepared composite, titanium-coated track-etched nanofiber membrane (TTM-CPnf) was characterized by Fourier transform infra-red (FTIR), water contact angle, and scanning electron microscopy (SEM) analyses. Smooth and uniform CS nanofibers with an average fiber diameter of $156.55 \mathrm{~nm}$ were produced from a 70/30 CS/PEO blend solution prepared from 92 wt. \% acetic acid and electrospun at $15 \mathrm{~cm}$ needle to collector distance with $0.5 \mathrm{~mL} / \mathrm{h}$ flow rate and an applied voltage of $30 \mathrm{kV}$ on the TTM-CPnf. Short (15 min) and long (72 h)-term solubility tests showed that after $3 \mathrm{~h}$, crosslinked nanofibers were stable in acidic $(\mathrm{pH}=3)$, basic $(\mathrm{pH}=13)$, and neutral $(\mathrm{pH}=7)$ solutions. The crosslinked TTM-CPnf material was biocompatible based on the low mortality of freshwater crustaceans Daphnia magna. The composite membranes comprised of electrospun nanofiber and TMs proved to be biocompatible and may thus be suitable for diverse applications such as dual adsorption-filtration systems in water treatment.
\end{abstract}

() 2020 Elsevier Ltd. All rights reserved.

\section{Introduction}

Population growth and industrialization have increased the demand for clean water [1]. Industrialization has introduced many different chemical substances into the surrounding environment in the form of metal ions or persistent organic compounds such as dyes, which can compromise human health [2]. To increase the supply of clean water, the purification of water sources is important, but finding suitable materials for diverse water purification applications remains a current challenge. Electrospun nanofibers are of low cost and possess unique properties such as high porosity with fine pores, high permeability, low basis weight, and high specific surface area to volume ratio [3,4], and these properties initiated the use of electrospun nanofiber membranes as novel materials for water treatment.

\footnotetext{
* Corresponding author.

E-mail address: pereaokola@gmail.com (O. Pereao).
}

Chitosan (CS) and some of its derivatives have been of interest in electrospinning due to their excellent physicochemical properties. CS is the natural polymer obtained from the partial deacetylation of crustacean waste and has anti-algal, anti-fungal, and anti-microbial properties [5,6]. CS can be used for the adsorption of toxic metals because of the presence of amine and hydroxyl functional groups [7] but the electrospinning of CS is extremely difficult and challenging due to the low chain flexibility, low mechanical properties, and high viscosity [8]. The mechanical properties of CS nanofibers can be better enhanced with the blending of CS with polymers such as nylon-6, poly(vinyl alcohol), poly(caprolactone), polyethylene oxide (PEO), cellulose, and poly(lactic acid) [9]. To increase the spinnability of CS in this study, it was blended with PEO, which is a bio/mucoadhesive, semi-crystalline, and biocompatible synthetic polymer because of its ability to form hydrogen bonds $[10,11]$.

Track-etched membranes (TMs) are produced by polymer film irradiation using highly ionizing particles and subsequent 
chemical etching. Polymers that are commonly used for membranes include polyimide, polyvinylidene fluoride, polyethersulfone, polyethylene, terephthalate, and polycarbonate [12]. Polymer films can be irradiated by either accelerated ions or fission fragments from a cyclotron to form the latent TM tracks. The track etching process converts the latent tracks into hollow channels. During chemical etching, and at a controlled $\mathrm{pH}$ and temperature, some chemical etchants like an alkaline solution, hydrogen peroxide, or acetic acid can be used to change the latent ion tracks into definite and controlled pore sizes to produce the TM. Several developed etching methods have allowed for the formation of cigar-like, funnel-like, conical, cylindrical, and other track-etched pore shapes [13]. Characteristics such as density, shape, and pore size can be changed in a controllable varied manner to yield membranes with the requisite transport and retention properties. TMs are available in the market and are used in various applications such as porous supports, model capillary systems, templates, and precise filters for the separation of nano- and micro-structured materials [12].

TMs can provide very distinctive advantages over conventional membranes because their structures can be determined precisely [13]. The combination of TMs and nanofibers to form a composite can become an effective way of preparing new materials with tailored properties [14]. Electrospun nanofibers have porous structures with good pore-interconnectivity and high surface-tomass (or volume) ratio while TMs contain nano- and macro-pores of diverse structures $[3,15]$. The synergy of combining electrospun nanofibers and TMs can thus offer unique properties for filtration and adsorption-based systems [16]. In a study by Li et al. [17], composite nanofiber membranes were obtained via electrospinning CS nanofibers on PET polyester scrim and were used for $\mathrm{Cr}(\mathrm{VI})$ chemical filtration. Another study by Podgórski et al. [18] also showed that fibrous filters made of nanofibers can be economic and very promising to improve filtration. However, there is still limited published studies demonstrating the use of composite electrospun nanofibers in dynamic adsorption, separation, and application processes.

Crosslinking can improve the mechanical and chemical stability of nanofibers in composite membranes. Crosslinkers are polymer stabilizing agents that act through the bonding and coupling of chains in the functional groups and thus prevent the polymer dissolution [19]. Glutaraldehyde (GA) has successfully been used to crosslink CS nanofibers. GA is a homo-bifunctional crosslinker that can react with CS through a Michael-type adduct with terminal aldehydes, which leads to the formation of carbonyl groups, or via Schiff base reaction that leads to an imine functionality [20]. The chemical stability of crosslinked electrospun nanofiber using GA has been reported for metal ion adsorption from an aqueous solution [21,22].

Daphnia magna (D. magna) has been used in ecotoxicological research and regulatory testing as a freshwater invertebrate and several features of the planktonic crustacean make it appropriate for testing toxicity in the laboratory [23]. Most toxicological research has been based on acute toxicity data with these aquatic crustaceans and based on effective concentration evaluation (EC50) for immobilization or lethal concentration (LC50) for mortality [24].

In this work, the parameters of the electrospinning process were optimized to achieve beadless CS nanofiber mats on the titaniumcoated TM used as the collector. A systematic investigation to understand the solubility and electro spinnability of chitosan/PEO (CS/ PEO) was performed. This study investigated the possibility of CS, a 'green' hydrophilic polymer, blended with PEO for electrospinning directly on a metallized TM as a conductive cathode and membrane support. We demonstrated the obtained composite membrane for biocompatibility using $D$. magna. Improving composite membranes could allow their use as affinity membranes for filtration/adsorption of targeted toxic metals or organic pollutants.

\section{Materials and methods}

\subsection{Materials}

CS $\left(M_{W}=200,000\right)$, degree of deacetylation (DDA) 80-85\%, and PEO $\left(\mathrm{M}_{\mathrm{w}}=300,000\right)$ were used to prepare the nanofiber mats. Glacial acetic acid ( $\geq 99 \%$ ) was purchased from AppliChem (Germany). Commercial CS was purchased from Bioprogress (Russia). PEO was obtained from Sigma-Aldrich (Germany). GA solution grade II $25 \% \mathrm{w} / \mathrm{v}$ was obtained from Sigma Aldrich (USA). The Milli$\mathrm{Q}$ deionized water-18 $\Omega$ was used throughout this study from the Brand Millipore system Milli-Q France.

\subsection{Ti-thin-film track-etched membrane}

The TMs used in the study were obtained from the Joint Institute for Nuclear Research, Dubna, Russia using a Mitsubishi PET polyester film to obtain $23 \mu \mathrm{m}$-thick fabricated TMs having a pore density of $2.4 \times 10^{8} \mathrm{~cm}^{-2}$ with characteristic pore diameters of $0.3 \mu \mathrm{m}$. Different pore sizes can be achieved with TMs and this is just a typical example. The description of the TM fabrication process was detailed elsewhere [25], where irradiation was done by a cyclotron with the use of swift heavy ions $(\mathrm{Xe}, \mathrm{Kr})$ to create latent ion tracks on a PET film. These latent ion tracks were subsequently etched in $\mathrm{NaOH}(2 \mathrm{M})$ to produce the pores. The track-etched PET membranes were thereafter made conductive by using a magnetron sputtering technique to deposit thin films of Ti on the membrane. Integrating the Ti-based cathode into the TM improved the adhesion between electrospun nanofibers and the TM substrate and subsequently promoted larger scale electrospinning [26]. The metallization of the TM with Ti was used to prepare the TM as a cathode for use during electrospinning. The selection of titanium (Ti) as the coating was due to its good electrical conductivity, ease of production by magnetron sputtering, stability in water, and biological inertness. Copper and silver were considered as alternative conductive coatings but were deemed to be less effective based on other studies [15]. The adhesion between copper and the TM surface reduced considerably when in contact with water and silver was less stable and more expensive than titanium. The Ti metallic coatings were deposited from a vertically mounted target, which sputtered $99.7 \%$ pure titanium from an argon gas atmosphere (99.99\%) carried out in an industrial DC planar magnetron sputtering vacuum unit for roll-to-roll deposition. Glow discharge air plasma was used to clean the samples before depositing the Ti coatings on top of the TM for about a minute to improve the thinfilm adhesion to the polymer surface [27].

\subsection{CS solution preparation}

The electrospinning solution was prepared by dissolving CS $(0.75 \mathrm{~g})$ and PEO $(0.75 \mathrm{~g})$ separately using $92 \mathrm{wt}$ \% acetic acid (1 M) aqueous solution to achieve $5 \%(\mathrm{w} / \mathrm{v})$ polymer concentration. Then, an appropriate quantity of CS solution, which was prepared as described above, was added to PEO solutions at the following different blending ratios of 90/10, 70/30, 50/50, 30/70, and 10/90 (total volume $20 \mathrm{~mL}$ ) at room temperature. Separate solutions of $3.5 \mathrm{wt}$ \% and $7.5 \mathrm{wt}$ \% of CS dissolved in acetic acid (92 wt. \%) were also prepared individually. The blended mixtures were stirred on an IKA magnetic stirrer (RCT basic) consistently for at least $24 \mathrm{~h}$ for a complete dissolution of the polymers.

\subsection{Direct electrospun fibers on metallized TMs}

Unlike classical electrospinning, where a needle anode and collector-plate cathode are used [28], the collector-plate was 
integrated into the TM membrane by sputter coating metallic Ti onto the track membrane sheet before electrospinning. The prepared CS/PEO polymer blend solutions were loaded into a plastic $5 \mathrm{~mL}$ syringe before a metallic needle tip was attached. The syringe was then positioned in a ZS100 CR programmable pump to regulate the solution flow rate. Thereafter, a high voltage of between 15 and $30 \mathrm{kV}$ was applied to the metallized TM collector and the syringe needle. The voltage was applied while maintaining a pumping speed of $0.5 \mathrm{~mL} / \mathrm{h}$ to deposit the fibers, using a needle tip to the collector distance of $10-25 \mathrm{~cm}$. The solution was electrospun for a minimum time of $2 \mathrm{~h}$. The composite nanofiber mats were then carefully positioned above a porous Teflon plate inside an airtight desiccator which contained $10 \mathrm{~mL}$ of $25 \%$ aqueous GA solution in a petri dish beneath the Teflon plate to crosslink the fibers at $37^{\circ} \mathrm{C}$. After the crosslinking procedure, the composite nanofiber membrane samples were vented in a fume cupboard for an hour to eliminate any residual GA and were subsequently dried for $24 \mathrm{~h}$ in a vacuum.

\subsection{Solubility estimation test}

A modified procedure of the stability of the nanofiber under acidic, neutral, and basic conditions was tested as described by Kiechel and Schauer [29] and Austero et al. [20]. The TTM-CPnf nanofiber membranes cut into $7 \mathrm{~mm}$ by $7 \mathrm{~mm}$ square shapes were inserted in separate $15 \mathrm{~mL}$ solutions of different $\mathrm{pHs}$ with each containing $1 \mathrm{M}$ acetic acid (AA) of $\mathrm{pH} 3, \mathrm{H}_{2} \mathrm{O}$ of $\mathrm{pH}$, and $1 \mathrm{M} \mathrm{NaOH}$ of $\mathrm{pH} 13$ in a centrifuge tube. The transmittance at $600 \mathrm{~nm}$ (T600) of each solution was measured using a Helios Epsilon Spectrometer from Thermo Fisher Scientific Inc., MA, USA to monitor the solubility of nanofibers. Solution aliquots were removed and transferred to cuvettes for measurement after $15 \mathrm{~min}$ and $72 \mathrm{~h}$ at ambient temperature. The sampled $1.5 \mathrm{~mL}$ volume previously removed was substituted back into the solution after each measurement and three trials were conducted for each sampling regime. For the reference values, $1 \mathrm{M} \mathrm{NaOH}, 1 \mathrm{M} \mathrm{AA}$, and $\mathrm{H}_{2} \mathrm{O}$ solutions were used and all the different test solutions were normalized to the corresponding reference solution. Each composite nanofiber membrane condition was also visually observed and recorded.

\subsection{Swelling of the composite nanofiber membrane}

The swelling ability of the crosslinked nanofiber membranes was investigated by immersing pre-weighed (approximately $0.05 \mathrm{~g}$ ) composite nanofiber in $\mathrm{pH} 7$ (neutral), $\mathrm{pH} 10.01$ (alkaline), and $\mathrm{pH} 4.01$ (acidic) solutions ( $10 \mathrm{~mL}$ volume) for $12 \mathrm{~h}$ and $24 \mathrm{~h}$ at room temperature. Thereafter, the excess solvents were removed by blotting on a filter paper and the samples were weighed using an analytical balance OHAUS Adventurer (USA). The swelling rate of the composite nanofibers was evaluated using the following equation

Degree of swelling $(\%)=\left(\frac{S_{w}-D_{w}}{D_{w}}\right) \times 100$

where $D_{w}$ is the initial dry weight of the nanofiber membrane and $S_{w}$ is the weight of the swollen nanofiber membrane.

\subsection{Characterization}

The infrared spectra of the nanofiber mats were measured using a Fourier transform infrared spectrometer fitted with an attached attenuated total reflectance (FTIR-ATR) (Thermo Scientific model,
Nicolet 6700). The spectra were collected in the $4000-500 \mathrm{~cm}^{-1}$ range with a $4 \mathrm{~cm}^{-1}$ resolution by the accumulation of 64 scans to confirm the hydrogen bonding formation between CS and PEO and to observe the chemical functional groups on the fibers. The electrospun nanofiber (non-cross-linked, cross-linked electrospun CS/ PEO) mats as well as the surface layer microanalysis of the polymer thin film TM-Ti structures was evaluated with a SU-8020 fieldemission scanning electron microscope (Hitachi-Japan) (FESEM). The samples were coated with palladium-platinum for $5 \mathrm{~s}$ before microscopy using a Denton vacuum desk II sputtering machine. The average fiber diameter distribution was estimated by measuring 50 random fibers from the FESEM acquired images using an image $\mathrm{J}$ software analysis (National Institute of Health, USA, Image J). Elemental distributions were obtained by energy dispersive spectroscopy (EDS), performed on the FESEM with the Oxford Instruments Aztec Energy EDS Analysis System. The different polymer solutions' electrical conductivity was tested using a Mettler Toledo (USA) Seven2Go S3-Basic conductivity portable meter and the measurements were reported after five replications at $25{ }^{\circ} \mathrm{C}$. A Krüss drop shape analyzer-DSA100S (KRÜSS $\mathrm{GmbH}$-Germany) was used at room temperature to measure the water contact angle where $4 \mu \mathrm{L}$ drop of ultra-pure water was released over the sample from a needle and observed through a video camera, which also allowed for the exact capturing of the moment when the drop touched the sample surface.

\subsection{Assessment of the biocompatibility potential of the composite}

A $0.01 \mathrm{~g}$ of the composite nanofiber membrane of TTM-CPnf-2 and GA-modified TTM-CPnf-2 was weighed, immersed, and shaken by sonication for approximately $30 \mathrm{~min}$ in $100 \mathrm{~mL}$ Milli-Q water to achieve $100 \mathrm{mg} / \mathrm{L}$ 'stock suspension'. The Daphnia acute toxicity testing was carried out using the suspension (OECD guideline 202) and ISO norm 6341 [30] as previously described by Heinlaan et al. [31]. Hatching of the ephippia was achieved according to the supplier's (Daphtoxkit F Magna ${ }^{\mathrm{TM}}$, MicroBioTests Inc., Belgium) instructions. The young daphnids were pre-fed with a suspension of Spirulina microalgae $\left(6.7 \times 10^{4}\right.$ cells $\left./ \mathrm{mL}\right) 2 \mathrm{~h}$ before the commencement of experiments to prevent 'starvation to death'. Hatched ephippia were used for the toxicity testing as daphnid neonates, which are $<24 \mathrm{~h}$ old and incubated at $20{ }^{\circ} \mathrm{C}$ under the illumination of 6000 Lux continuously for up to $96 \mathrm{~h}$. A control (no TTM-CPnf composite) and at least four technical replicates and three independent assays were conducted for concentrations of $6.25,12.5,25,50$, and $100 \mathrm{mg} / \mathrm{L}$. Testing was done in an Artificial Freshwater (AFW) OECD 202 medium composed of $294 \mathrm{mg} / \mathrm{L}$ $\mathrm{CaCl}_{2} \cdot 2 \mathrm{H}_{2} \mathrm{O} ; 67.75 \mathrm{mg} / \mathrm{L} \mathrm{NaHCO}{ }_{3} ; 123.25 \mathrm{mg} / \mathrm{L} \mathrm{MgSO} 4 \cdot 7 \mathrm{H}_{2} \mathrm{O}$; and $5.75 \mathrm{mg} / \mathrm{L} \mathrm{KCl}$ at $\mathrm{pH} 7.8 \pm 0.2$ of ultrapure water in MicroBio Tests, Inc. (Mariakerke-Gent, Belgium) polycarbonate 30-well test plates with each $10 \mathrm{~mL}$ well containing 5 daphnids in the test wells. AFW are synthetic solutions that emulate the major ion compositions of natural waters that are important in experiments for the rearing of stock cultures and have the advantages of standardization and reproducibility [32]. The immobilization (mortality) by visual observation of daphnids was recorded with the use of a light table upon 24 and $48 \mathrm{~h}$ incubation in the dark at $20^{\circ} \mathrm{C}$. The daphnid was considered as immobilized if swimming was not resumed after $15 \mathrm{~s}$ of gentle agitation. The EC50 toxicity endpoint was considered as the toxicant concentration that was required for immobilizing 50\% daphnids after the exposure time. The ToxRat Professional $3.2 ®$ software was used for the determination of mortality, statistical significance, and critical concentrations. The test was considered valid if the control daphnids immobilization did not exceed $10 \%$. Low toxicity would indicate biocompatibility. 


\section{Results and discussion}

\subsection{SEM-EDS analyses of TTM structures}

The titanium-coated polyethylene terephthalate track-etched membranes (TTM) surface structure has a typical surface for a track membrane as shown in Fig. 1(a) and a single pore track in (1b) while the elemental composition of a $\mathrm{Ti}$ film deposited by magnetron sputtering from EDS quantitative results is shown in Table 2 indicating the Ti peaks.

The pores of TTM have a symmetrical circular shape with a diameter of $0.2 \mu \mathrm{m}$ providing a high flow rate. Energy-dispersive microanalysis confirmed the titanium layer (titanium -1.41\%) on the TTM after sputtering. The presence of elements such as silicon and aluminum can be explained as the technological feature of working with the sample (aluminum stage, particles of silicon and carbon, in the form of surface contamination) while the occurrence of oxygen indicated the oxidation surface, which formed the $\mathrm{Ti}_{1-\mathrm{x}} \mathrm{O}_{\mathrm{x}}$ dielectric layer.

The SEM-EDS quantitative result showed that the carbon content is due to PET while oxygen and titanium contents are due to the $\mathrm{TiO}_{2}$ used in covering the surface of the TM from the Ti film deposited by magnetron sputtering.

\subsection{Properties of blend electrospinning solutions}

\subsubsection{Electrical conductivity}

The polymer blends were investigated for electrical conductivity using the conductivity portable meter and Fig. 2 shows the trend of electrical conductivity in the different blend ratios of $5 \mathrm{wt} . \% \mathrm{CS} / \mathrm{PEO}$ prepared using acetic acid solutions (see Table1). The electrospinning process can be affected by the electrical conductivity of solutions and the conductivity of CS/PEO blended solutions increased from 55 to $944 \mu \mathrm{S} / \mathrm{cm}$ with the increase of CS ratio because PEO is a non-ionogenic polymer and CS is a polyelectrolyte. Electrospinning CS singly was difficult because solutions of CS are more conductive than PEO because of the positive charges and polycationic nature of the polymer chains. The PEO addition decreased the CS solution's electrical conductivity by the reduction in the protonation amount due to the formation of hydrogen bonds occurring between the ether groups of PEO and amino groups of CS, and also by the substitution of a positively charged molecule by a neutral one, which thus helped to obtain a stable needle jet and prevented the jet splaying in the region of the stretch $[33,34]$.

The hydrogen bond formation between CS amino hydrogen and PEO polyether oxygen rendered CS more electro spinnable and increased the solution chain entanglements [35]. Though some
Table 1

Sample code and polymer ratio (5 wt. \% blend CS/PEO ratios 100/0, 90/10, 70/30, 50/50, $30 / 70,10 / 90$, and 0/100)

\begin{tabular}{llll}
\hline Sample code & Polymer & Polymer Ratio & Polymer to solvent $(\%)$ \\
\hline TTM-C-nf & CS & 100 & $5 \mathrm{wt}$. \\
TTM-CPnf-1 & CS/PEO & $90 / 10$ & $5 \mathrm{wt}$. \\
TTM-CPnf-2 & CS/PEO & $70 / 30$ & $5 \mathrm{wt}$. \\
TTM-CPnf-3 & CS/PEO & $50 / 50$ & $5 \mathrm{wt}$. \\
TTM-CPnf-4 & CS/PEO & $30 / 70$ & $5 \mathrm{wt}$. \\
TTM-CPnf-5 & CS/PEO & $10 / 90$ & $5 \mathrm{wt}$. \\
TTM-P-nf & PEO & 100 & $5 \mathrm{wt}$. \\
\hline
\end{tabular}

Table 2

Energy-dispersive quantitative analysis of the elemental composition of Ti metallized thin film deposited by magnetron sputtering on TM.

\begin{tabular}{ll}
\hline Element & Atomic \% \\
\hline $\mathrm{C}$ & 95.33 \\
$\mathrm{O}$ & 2.82 \\
$\mathrm{Al}$ & 0.34 \\
$\mathrm{Si}$ & 0.1 \\
$\mathrm{Ti}$ & 1.41 \\
Total & 100 \\
\hline
\end{tabular}

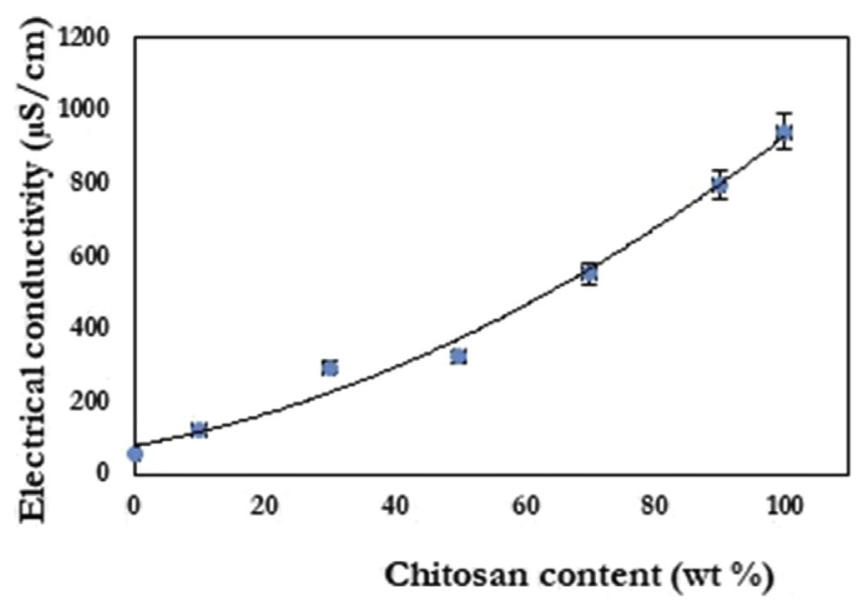

Fig. 2. The effect of electrical conductivity on the CS/PEO composite solution blends (blending ratios-100/0, 90/10, 70/30, 50/50, 30/70, and 10/90 and 0/100; 5 wt. \% CS/ PEO polymer solution in $92 \mathrm{wt}$ \% acetic acid; temp $37^{\circ} \mathrm{C}$ ).
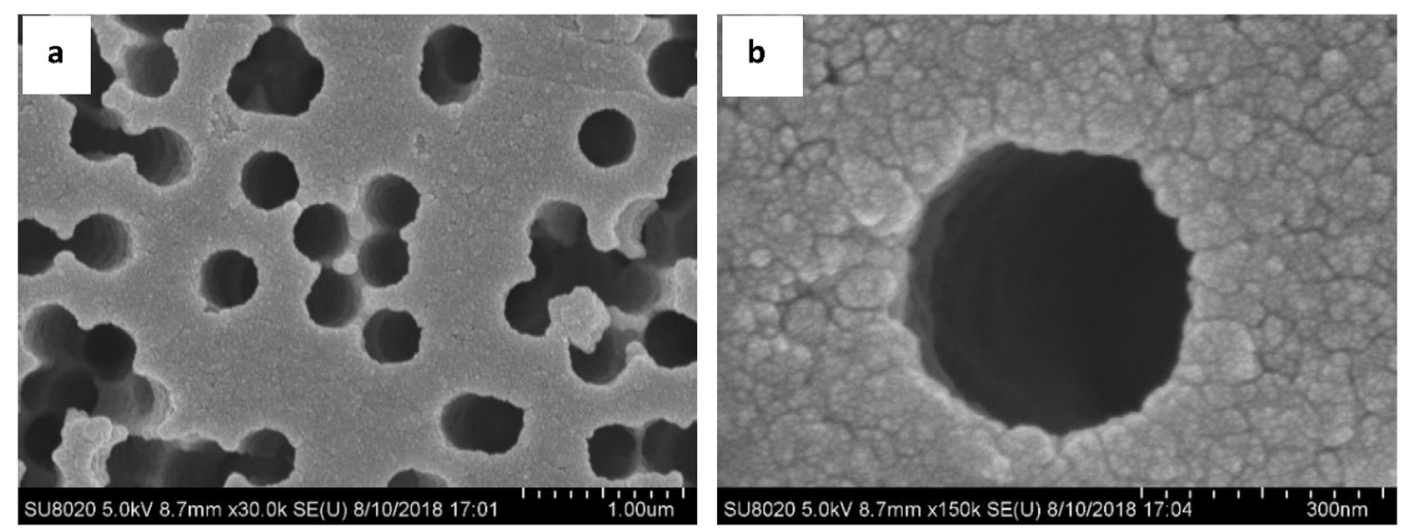

Fig. 1. a) SEM micrograph of a Ti film deposited by magnetron sputtering TMs and b) typical pore track of the TMs. 


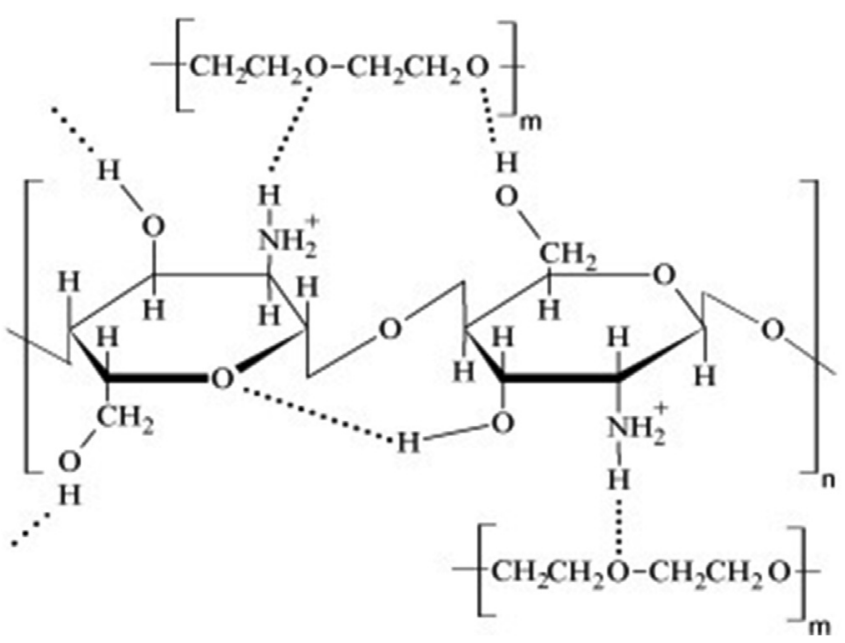

Fig. 3. Proposed schematic of the chemical structures of CS/PEO interactions.

applications may require a CS specific property like adsorption and filtration, the lowest added 30\% ratio of PEO polymer was preferable, because the PEO addition lowered the electrical conductivity sufficiently without altering the outstanding electrospinnability. The high CS content of CS-based nanofibers was achieved previously, but quite a lot of these studies were based on the use of other solvents like dimethyl sulfoxide and trifluoroacetic acid [36]. However, in this study, TTM-CPnf mats with high CS content were electrospun using acetic acid aqueous solutions, which presents a green option.
CS and PEO represent a blended mixture from two structurally different polymers that interact together without any covalent bond formation. It was already demonstrated that after CS/PEO electrospinning, PEO can be removed subsequently from the nanofibers by simply incubating the CS/PEO nanofibers in water for a few days [37,38]. Many nanofibers of PEO and CS blends have been fabricated by electrospinning based on the interaction between PEO moieties and CS chains [39], as shown in Fig. 3, which enabled flexible PEO chains to easily form molecular complexes through hydrogen bonding between CS and PEO.

Two types of hydrogen bonds can be formed between CS and PEO from the proposed schematic: the first may occur between the ether oxygen of PEO and the hydrogen atom of the quaternary ammonium group, while the second type is between the hydrogen atoms of hydroxyl groups of CS and ether oxygen of PEO [39].

\subsection{Scanning electron microscopy of nanofiber structures}

The SEM micrographs of 3.5 wt. \%, 5 wt. \%, and 7.5 wt. \% polymer solutions of CS and 5 wt. \% PEO prepared in mixed distilled water and $92 \mathrm{wt} . \%$ acetic acid solution without the addition of any blend polymers are shown in Fig. 4. The TTM-C-nf (CS only) resulted in a very high solution viscosity and thus prevented the solution elongation from the needle jet in the electrical field of the electrospinning unit (Fig. 4(a)) but TTM-P-nf (PEO only) solution was electrospun (Fig. 4(b)) and produced nanofibers. It was therefore considered as an appropriate blending polymer for the nanofiber fabrication in this study. The results showed that TTM-C-nf (Fig. 4(a)) and TTM-P-nf (Fig. 4(b)) have average fiber diameters (AFDs) of $157.074 \pm 12$ and $181.59 \pm 12$ respectively, but the TTM-C-
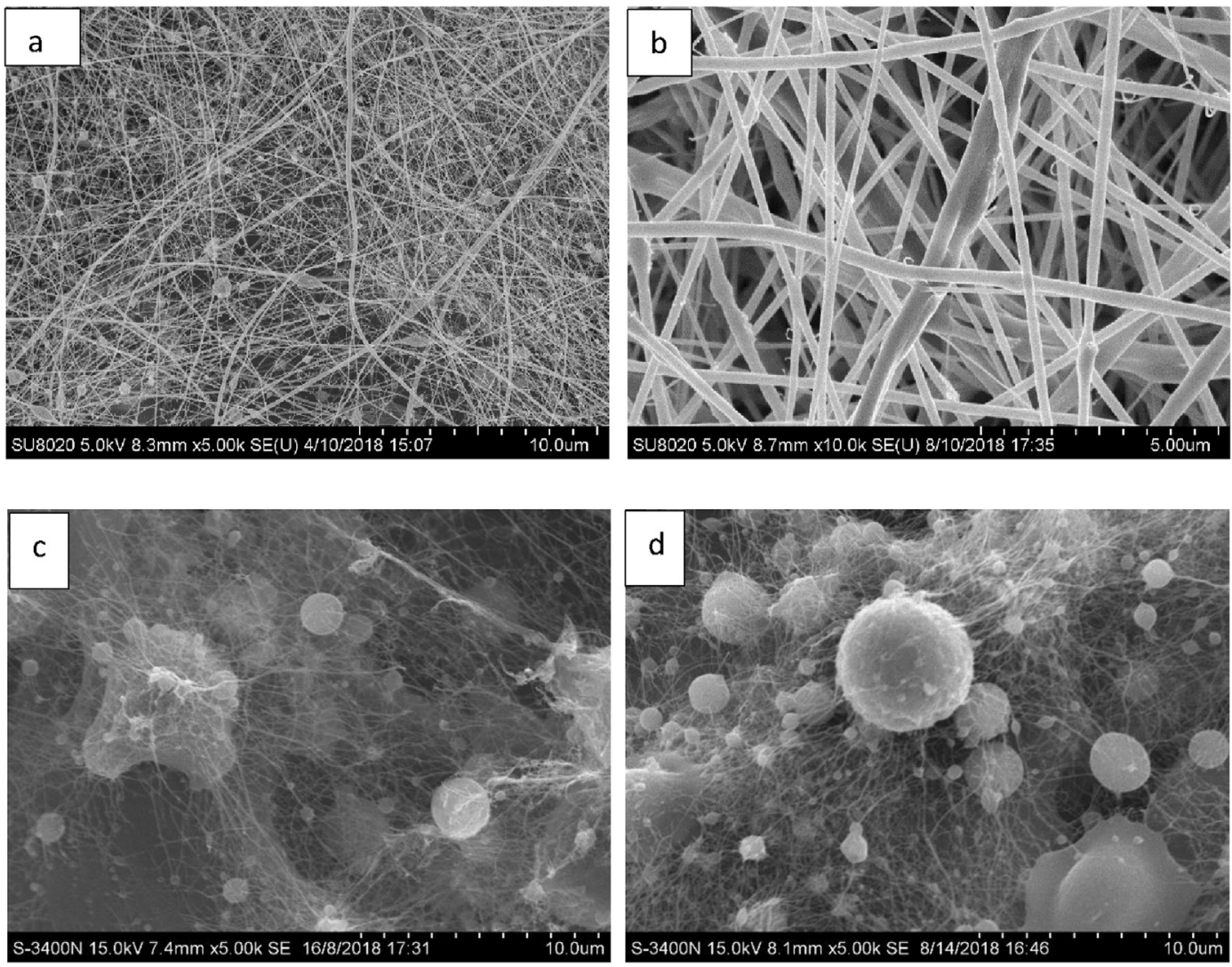

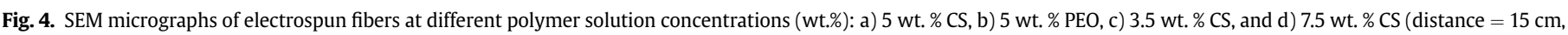
voltage $=30 \mathrm{kV}$, flow rate $=0.5 \mathrm{~mL} / \mathrm{h}$, solvent $=92 \mathrm{wt}$. \% acetic acid; temp $37^{\circ} \mathrm{C}$ ). 

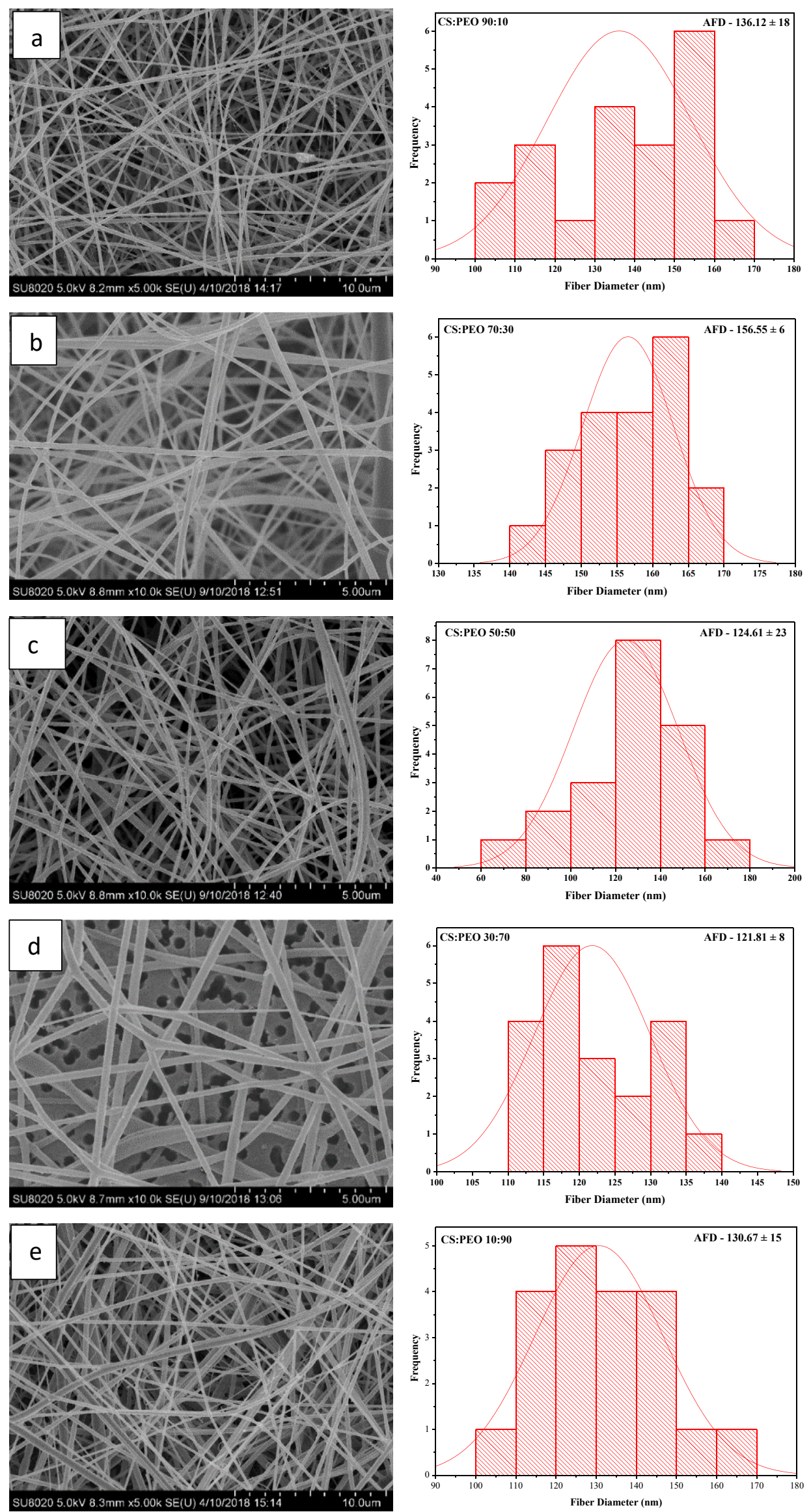
nf in Fig. 4(a) had a substantial quantity of elongated beads deposited on the collector. Clean PEO nanofiber was produced and used as a control to compare with the fibers obtained from different CS wt \% to evaluate the preliminary morphology of fabricated nanofibers. In the case of $3.5 \mathrm{wt}$ \% (Fig. 4(c)), the solution produced blobs at the tip of the needle before being drawn out into the nanofiber form probably due to high surface tension and lack of chain entanglements, but when the CS content was increased to 7.5 wt. \% (Fig. 4(d)), uneven beaded nanofibers were obtained, which could be attributed to the increase in viscosity [34]. Hence, the $5 \mathrm{wt}$ \% CS content, which produced uniform electrospun nanofibers with average diameter, can be used for creating smooth nanofibers.

Beads were noticed in several cases despite changes in various CS electrospinning parameters including flow rate and voltage. As shown in Fig. 4(b), TTM-P-nf was able to form nanofibers by itself and can thus improve the electrospinning process. Though there was a noticeable improvement in the PEO spinning process, it was difficult to spin for longer times beyond $1 \mathrm{~h}$. This result is similar to the electrospinning of CS using an acetic acid solution by Geng et al. [40] who observed that when the CS concentration was increased at the same equal molecular weight, there was a gradual morphological change from a mixture of the droplet and nanofiber, to uniform nanofibers to nanofibers having spindle-like beads. Therefore, the ratios of the CS and PEO polymer solutions were varied at different CS/PEO percentages for an improvement in the electrospinning and mechanical properties of CS nanofibers. Fig. 5 shows the SEM images of 5 wt.\% CS/PEO having different blending ratios of 90/10, 70/30, 50/50, 30/70, and $10 / 90$.

The results in Fig. 5(a-e) indicate that the PEO improved the electrospinning of CS when compared with Fig. 4(a). These results compared favorably with results obtained by Han et al. [27], when he used PEO as a partner polymer for the electrospinning of methoxy poly(ethylene glycol)-grafted CS. From this investigation, the CS/PEO ratios of 90/10 (TTM-CPnf-1) and 70/30 (TTM-CPnf-2) were deemed feasible and spinnable.

The AFDs Fig. 5(a-e) obtained are $136.12 \pm 18,156.55 \pm 6$, $124.61 \pm 23,121.81 \pm 8$, and $130.67 \pm 15$ for $90 / 10,70 / 30,50 / 50,30 /$ 70 , and 10/90 respectively. There was a decrease in AFD when PEO was initially added to the CS polymer solution but with very little effect for TTM-CPnf-2. However, diameters decreased with further addition until there was no significant AFD effect with additional PEO polymer. The effect of electrospinning process parameters, including screen distance and applied voltage, on the TTM-CPnf-2 nanofiber diameter, was further investigated to understand the electrospinning behavior of TTM-CPnf-2 as shown in Fig. 6 .

By increasing the tip-to-collector distance (Fig. 6(a)), the jet solution stability increased and gave rise to the formation of nonwoven homogeneous nanofiber mats with higher diameters, but an increase in applied potential (Fig. 6(b)) made the fibers more cylindrical and reduced bead formation [41]. Moreover, while the nanofibers' average diameter decreased, the morphology of the electrospun nanofibers was improved and there was no fiber alignment due to the increased electric field strength despite increasing the jet solution instabilities. However, nanofibers prepared by Singh et al. (2020) [42] using a similar process as this study with little modifications showed that through an increase of voltage from $17.5 \mathrm{kV}$ to $22.5 \mathrm{kV}$, the average fiber diameter of their $\mathrm{CS} / \mathrm{PEO}$ nanofibers increased from $108 \mathrm{~nm}$ to $149 \mathrm{~nm}$. Fig. 7 depicts the Ti-coated track-etched membrane (TTM) before electrospinning, after electrospinning (TTM-CPnf-2), after crosslinking with GA (TTM-CPnf-2G), and cross-section showing the nanofiber adhesion on the TM.

The SEM micrographs in Fig. 7(b) indicate that the CS/PEO composite nanofibers covered the surface of the TTM in Fig. 7(a) through the electrospinning process and the nanofibers can be observed above the TTM pores. Fig. 7(b) and (c) also shows the hierarchical pore structure dimensions formed by the nanofiber-interfiber voids on top of the TTM. Fig. 7(c) micrographs show cross-linked fibers after the GA treatment leading to nanofibers with a cross-linked matrix while Fig. 7(d) shows a crosssection of the nanofiber adhering to the top of the membrane. The nanofibers were smooth and straight before the treatment, but after the modification, the nanofibers became entangled and interlocked by crosslinking. The interfiber voids in Fig. 7(b) were quite large but the voids reduced to a smaller pore space after the crosslinking as shown in Fig. 7(c). A contraction and consequent thickening and increase of the fiber diameters were noticed without losing their cylindrical shape. These mechanical interlocks helped to prevent nanofiber delamination from the support as seen in Fig. $7(\mathrm{~d})$.

The prepared nanofibers were similar to the CS/polyethylene oxide (PEO) electrospinning blend process reported by Spasova et al. [43], where CS was prepared in $0.3 \mathrm{M}$ acetic acid while PEO was prepared using distilled water and the electrospinning of the blend was only feasible in a CS/PEO mass ratio of $<5$, but achieved without a membrane support. Studies by Bhattarai et al. [44] reported the CS/PEO blend electrospinning, which showed that the CS nanofibers formation was strongly dependent on the mass ratio blend of CS/PEO. Additionally, Ignatova et al. [45], showed that the preparation of a polyelectrolyte like CS in an aqueous solution by electrospinning was possible in the presence of an additional second polymer only. The formation via electrospinning of composite CS-based nanofiber on TTM using a biodegradable polymer blend (CS/PEO) is being reported here for the first time. The nanofibers and TMs were combined to form a composite nanofiber TM.

\subsection{Fourier transform infrared spectroscopy}

The observed chemical structure and functional groups on TTMC-nf, TTM-P-nf, and TTM-CPnf blend in distilled water and $92 \mathrm{wt} . \%$ acetic acid solution as determined by FT-IR are shown in Fig. 8. The ether, amine $\left(\mathrm{NH}_{2}\right)$, carbonyl (CO-NHR), and hydroxyl groups in CS all form inter/intrachain hydrogen bonds [46]. The hydrogen bonding between the amino and hydroxyl groups on the ether groups in PEO and CS molecules is considered as part of the key reason for the electrospinning [39,46]. The observed vibrational peak at $1112 \mathrm{~cm}^{-1}$ is a characteristic of the adsorption vibrational stretch of the ether group $(\mathrm{C}-\mathrm{O}-\mathrm{C})$ [47]. This peak shifted to a lower wavenumber gradually with an increase in the CS content in the blended nanofibers.

The FTIR spectra obtained for TTM-C-nf and TTM-CPnfs blended at different $\mathrm{CS} / \mathrm{PEO}$ percentage ratios showed a broad vibrational peak at $1614 \mathrm{~cm}^{-1}$ in the stretch amine $\left(\mathrm{NH}_{2}\right)$ region, which can be ascribed to the amine band in CS [48], but this peak steadily reduced with an increase in the PEO content towards TTM-P-nf. The CS (Fig. 8a) broad band at $1614 \mathrm{~cm}^{-1}$ is usually ascribed to the $\mathrm{N}-\mathrm{H}$ and $\mathrm{O}-\mathrm{H}$ stretching of the primary amino groups because of the hydrogen bonds with $\mathrm{O}-\mathrm{H}$ groups and $\mathrm{N}-\mathrm{H}$ stretching of the secondary amides [49]. The shift in the amine peak was visible, but after 50\% PEO addition to the nanofiber blend, this peak disappeared (Fig. 8(c). The FTIR absorption of PEO (Fig. 8e) showed its featured bands, which are assigned to the $\mathrm{CH}_{2}$ stretching around 
a

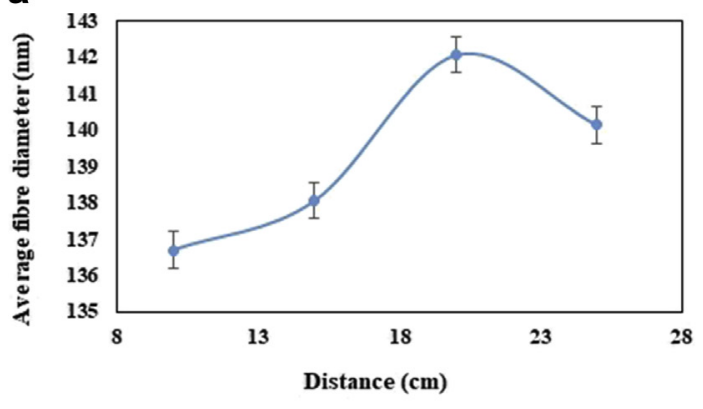

b

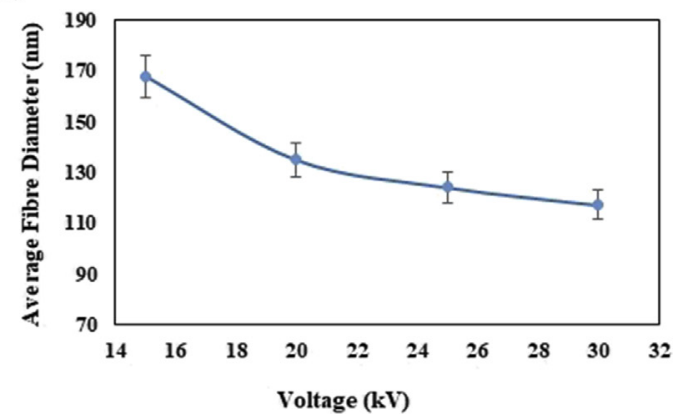

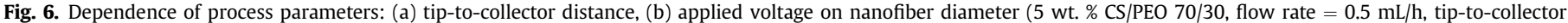
distance: (a) $10 \mathrm{~cm}$, (b) $15 \mathrm{~cm}$, (c) $20 \mathrm{~cm}$, and (d) $25 \mathrm{~cm}$; applied voltage: (a) $15 \mathrm{kV}$, (b) $20 \mathrm{kV}$, (c) $25 \mathrm{kV}$, and (d) $30 \mathrm{kV}$; solvent = $92 \mathrm{wt}$. \% acetic acid; temp $37{ }^{\circ} \mathrm{C}$ ).

$2876 \mathrm{~cm}^{-1}$, but this band overlaps with the band observed for CS [8]. PEO has a typical triplet $\left(1144,1099\right.$, and $\left.1059 \mathrm{~cm}^{-1}\right)$ having a maximum at $1099 \mathrm{~cm}^{-1}$, which was ascribed to $\mathrm{C}-\mathrm{O}-\mathrm{C}$ vibration [50], and sharp peaks at 960 and $1098 \mathrm{~cm}^{-1}$ are ascribed to the PEO asymmetric stretching of $-\mathrm{C}-\mathrm{O}$ [51]. The PEO polyether groups form hydrogen bonds with CS and the shift in hydroxyl, amine, and ether bonds in the CS/PEO nanofibers may be ascribed to the creation of hydrogen bonds between polyether oxygen and amino hydrogen in CS and PEO respectively [46]. Stronger interactions between CS/PEO bonds may prevail due to the formation and interaction of the hydrogen bonding. The sharp transmittance peaks (Fig. 8b-d) at 1341 and $1062 \mathrm{~cm}^{-1}$ are attributed to the stretching vibration associated with $-\mathrm{C}-\mathrm{N}$ in the pyranose ring and
$-\mathrm{C}-\mathrm{O}-\mathrm{C}-$ vibration in the PEO structure [52]. The absorbance intensity ratio at 1466 and $1279 \mathrm{~cm}^{-1}$ can be used as an indication of the CS/PEO ratio. Generally, before crosslinking, the bond between the CS/PEO nanofibers and the TTM membrane was not durable and strong enough to allow for the application of the composite in water-based adsorption and filtration processes, and TTM-CPnf-2 was selected to demonstrate the effect of the GA crosslinking.

\subsection{Surface wettability test of crosslinked TTM-CPnf-2}

Cross-linking of CS/PEO-70/30\% (TTM-CPnf-2) with GA was considered so that electrospun CS/PEO composite nanofiber mats could be applied in a larger range of water-based applications and
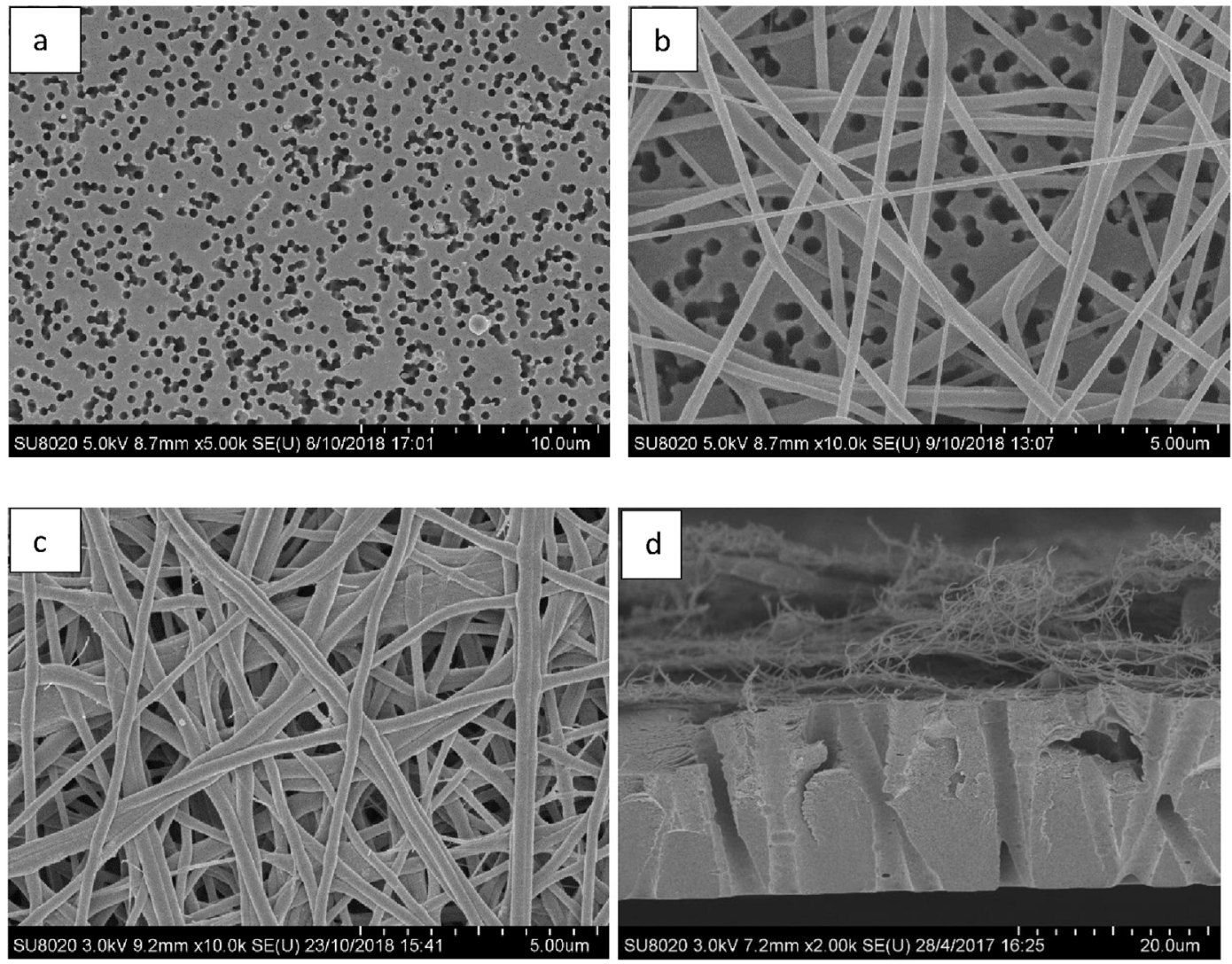

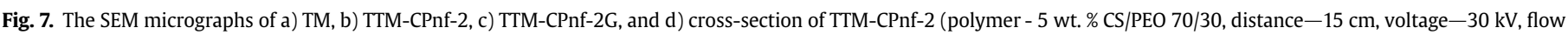
rate $-0.5 \mathrm{~mL} / \mathrm{h})$. 


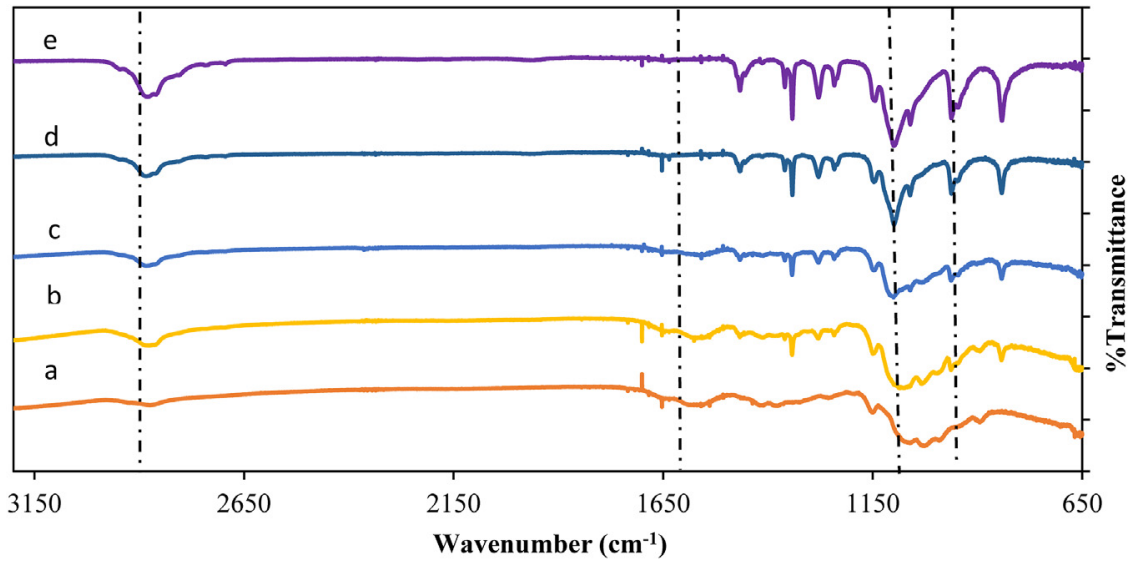

Fig. 8. FT-IR spectra of a) TTM-C-nf; b) TTM-CPnf-2; c) TTM-CPnf-3; d) TTM-CPnf-4; and e) TTM-P-nf (5 wt.\% polymer concentration, solvent-92 wt. \% acetic acid).
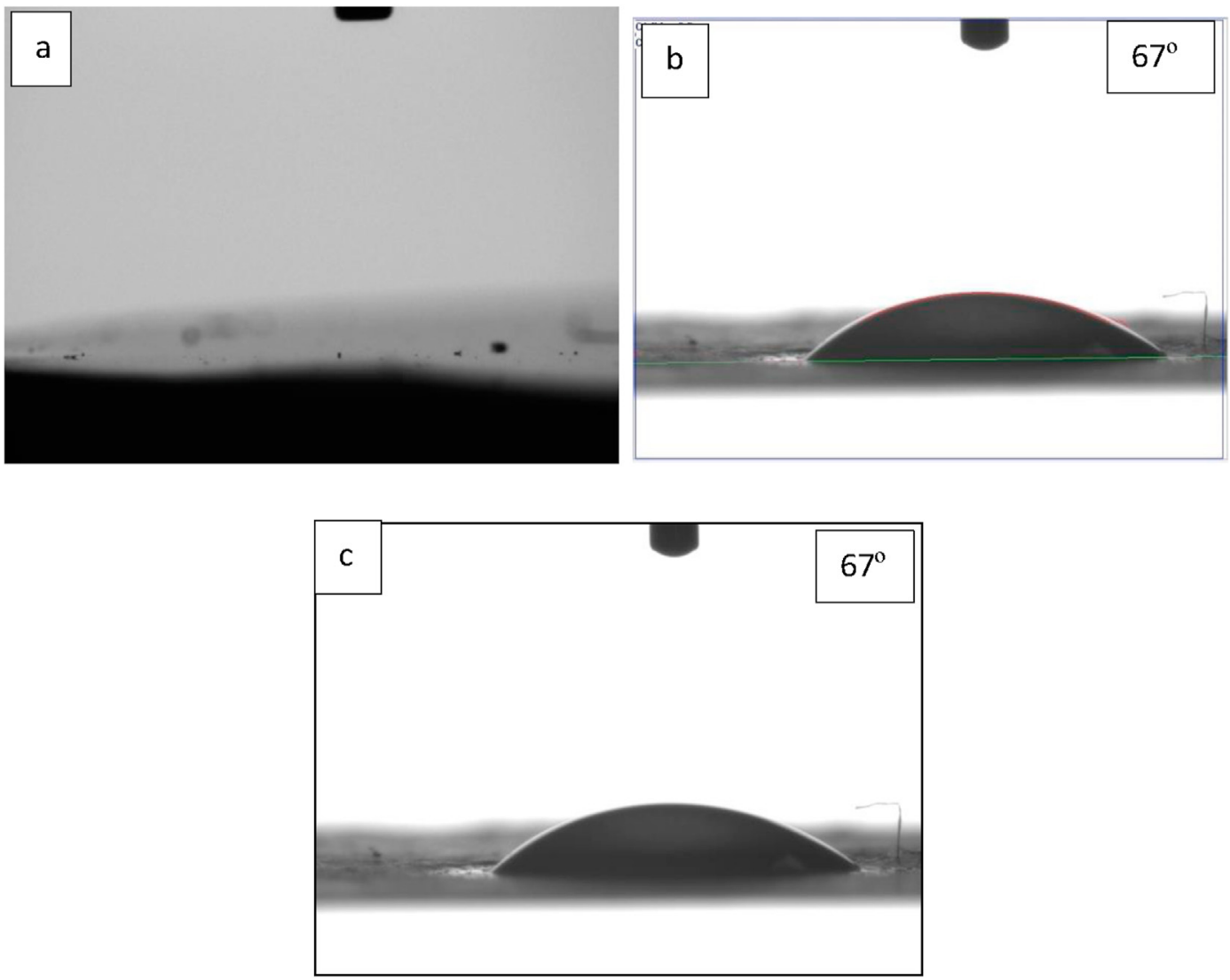

Fig. 9. Water drop images of (a) TTM-CPnf-2, (b) TTM-CPnf-2G, and (c) TTM-CPnf-2G after 30 min (polymer-5 wt. \% CS/PEO $70 / 30$, temp $37{ }^{\circ} \mathrm{C}$ ).

to have suitable structural integrity. To render TM-CPnf-2 slightly insoluble in water, GA vapor was investigated to crosslink the nanofibers. GA has high reactivity toward hydroxyl and amine groups and was chosen as the crosslinking agent [53]. GA vapor phase crosslinking can play an important part in the formation of water-stable nanofiber mats and thus improve their mechanical properties. The water contact angle was used as a measure of the wettability of electrospun TTM-CPnf-2 nanofiber mats after crosslinking using GA (TTM-CPnf-2G). Fig. 9 shows contact angle images of a pristine TM-CPnf-2 fabricated blend using noncrosslinked TM-CPnf (CS/PEO 70/30\%) and crosslinked TM-CPnf (TTM-CPnf-2G).
The surface wettability was investigated and observed with the introduction of some water droplets on the two different nanofiber mats. A water drop on the pristine TTM-CPnf-2 spreads immediately as shown in Fig. 8(a), demonstrating that water was immediately absorbed and no contact angle was visible, which indicates extreme hydrophilicity. Spontaneous water intrusion to the pore occurred without extra pressure to the TTM-CPnf-2 due to the high hydrophilic nature of the composite nanofiber membrane. GA cross-linked fibrous membranes improved the hydrophobic property of the TTM-CPnf-2 surface considerably to resist dissolution in water when compared to the non-crosslinked CS nanofiber, which dissolved in water. 
Table 3

Transmittance result of the visual observations of nanofiber solubility using TTM-CPnf-2G composite membrane in different $\mathrm{pH}$ solutions for 15 min and $72 \mathrm{~h}$.

\begin{tabular}{|c|c|c|c|c|c|c|c|}
\hline Solution & Mats & Crosslink time (hr) & $15 \min \mathrm{T} 600$ & Visual observation & 72 h T600 & Visual observation & Comments \\
\hline \multirow[t]{7}{*}{1 M AA pH 3} & & & 100 & & 98 & & \\
\hline & TTM-CPnf-2 & & 100.8 & $\mathrm{~N}$ & 99.6 & $\mathrm{~N}$ & Dissolved \\
\hline & TTM-CPnf-2G & 1 & 99.6 & $\mathrm{~N}$ & 96.2 & $\mathrm{~N}$ & Dissolved \\
\hline & TTM-CPnf-2G & 3 & 100.3 & $\mathrm{Y}$ & 95.6 & $\mathrm{Y}$ & Partially dissolved \\
\hline & TTM-CPnf-2G & 6 & 100.9 & $\mathrm{Y}$ & 97.1 & $\mathrm{Y}$ & Not dissolved \\
\hline & TTM-CPnf-2G & 12 & 100.1 & $\mathrm{Y}$ & 97.7 & $\mathrm{Y}$ & Not dissolved \\
\hline & TTM-CPnf-2G & 24 & 101 & $\mathrm{Y}$ & 99 & $\mathrm{Y}$ & Not dissolved \\
\hline \multirow[t]{7}{*}{$\mathrm{H}_{2} \mathrm{O} \mathbf{p H} 7$} & & & 99 & & 101 & & \\
\hline & TTM-CPnf-2 & & 100.6 & $\mathrm{~N}$ & 99.5 & $\mathrm{~N}$ & Dissolved \\
\hline & TTM-CPnf-2G & 1 & 101.2 & $\mathrm{~N}$ & 99.7 & $\mathrm{~N}$ & Dissolved \\
\hline & TTM-CPnf-2G & 3 & 101.1 & $\mathrm{Y}$ & 99.4 & $\mathrm{Y}$ & Not dissolved \\
\hline & TTM-CPnf-2G & 6 & 101.8 & $\mathrm{Y}$ & 99.5 & $\mathrm{Y}$ & Not dissolved \\
\hline & TTM-CPnf-2G & 12 & 102.1 & $\mathrm{Y}$ & 99.7 & $\mathrm{Y}$ & Not dissolved \\
\hline & TTM-CPnf-2G & 24 & 101.6 & $\mathrm{Y}$ & 99.8 & $\mathrm{Y}$ & Not dissolved \\
\hline \multirow[t]{7}{*}{1 M NaOHpH 13} & & & 101 & & 102 & & \\
\hline & TTM-CPnf-2 & & 100.5 & $\mathrm{Y}$ & 100.5 & $\mathrm{Y}$ & Not dissolved \\
\hline & STTM-CPnf-2G & 1 & 100.1 & $\mathrm{Y}$ & 100.3 & $\mathrm{Y}$ & Not dissolved \\
\hline & TTM-CPnf-2G & 3 & 100.2 & $\mathrm{Y}$ & 100.4 & $\mathrm{Y}$ & Not dissolved \\
\hline & TTM-CPnf-2G & 6 & 100.3 & $\mathrm{Y}$ & 100.2 & $\mathrm{Y}$ & Not dissolved \\
\hline & TTM-CPnf-2G & 12 & 100.4 & $\mathrm{Y}$ & 100.3 & $\mathrm{Y}$ & Not dissolved \\
\hline & TTM-CPnf-2G & 24 & 100.1 & $\mathrm{Y}$ & 99.9 & $\mathrm{Y}$ & Not dissolved \\
\hline
\end{tabular}

$\mathrm{N}=$ not visible; $\mathrm{Y}=$ visible.

Table 4

Average swelling values of TM-CPnf-2G for a period of 12 and $48 \mathrm{~h}$.

\begin{tabular}{lll}
\hline & Water sorption (\%) & \\
\cline { 2 - 3 } & $\mathrm{t}=12 \mathrm{~h}$ & $\mathrm{t}=48 \mathrm{~h}$ \\
\hline $\mathrm{pH} \mathrm{4.01}$ (acidic) & 85.72 & 82.14 \\
$\mathrm{pH} \mathrm{7} \mathrm{(neutral)}$ & 76.47 & 76.92 \\
$\mathrm{pH} \mathrm{10.01} \mathrm{(alkaline)}$ & 69.23 & 69.24 \\
\hline
\end{tabular}

The measured contact angle was $67^{\circ}$ for TTM-CPnf-2G. The crosslinked TTM-CPnf-2G was observed to be water-resistant and maintained the fiber morphology even after contacting the water drop for up to $30 \mathrm{~min}$ (Fig. 8(c)). This result showed that the crosslinked CS/PEO nanofiber on the TTM turned out to be more hydrophobic after incorporating the GA into the CS/PEO nanofibers on the TTM. A moderate water-resistance of nanofiber property is required in their use for adsorption and filtration applications despite the typical CS biodegradability in water. This measurement is a widely used method for evaluating the hydrophilicity/hydrophobicity where relatively small contact angles correspond to moderately hydrophilic surfaces [54]. The precise crosslinking reaction mechanism between CS and PEO blend is not well defined and it is not known whether the polymeric chains are linked via a chemical reaction between the two different polymers or between the crosslinking agent and the polymer. However, this polymer can complex with CS because of $\mathrm{C}-\mathrm{O}$ bonds in the PEO structure. CS can thus act as a proton donor while PEO can act as a proton acceptor, to yield homogeneous polymeric blends [55].

\subsection{Solubility results}

The transmittance (T600) and visual observations of a GAmodified composite nanofiber membrane, 5 wt. \% CS/PEO-70/30\% blend (TTM-CPnf-2G) soluble in $1 \mathrm{M} \mathrm{NaOH}, 1 \mathrm{M}$ Acetic acid (AA), and $\mathrm{H}_{2} \mathrm{O}$ respectively for $15 \mathrm{~min}$ and $72 \mathrm{~h}$, were investigated and are shown in Table 3.

The resistance to dissolution of the blended CS/PEO nanofiber was improved over an extensive $\mathrm{pH}$ range by crosslinking and the stability was tested in different $\mathrm{pH}$ solutions. The nanofibers were considered partially dissolved when the transmittance of the aliquot sample from the immersed TTM-CPnf composite membrane was greater than 50 . However, when the transmittance was greater than 90 but the nanofiber was not visually present in the solution, the nanofiber was considered as fully dissolved, but when the T600 was greater than 90 and the nanofiber can be visually seen or observed in the solution, then the nanofiber was considered not to be dissolved.

An improvement in the water stability of the nanofibers on the TTM-CPnf-2 composite membrane was visually detected after the crosslinking procedure. From neutral to the acidic $\mathrm{pH}$, CS was soluble in the solution because of the amine (pKa 6.3) protonation, until the functional groups were modified, or the polymer was crosslinked. The composite nanofibers were not soluble in $1 \mathrm{M}$ $\mathrm{NaOH}$ due to the amine group neutralization at high $\mathrm{pH}$ in CS [56]. GA crosslinking was able to prevent the dissolution of the nanofibrous structures of the composite membranes at lower $\mathrm{pH}$ after $3 \mathrm{~h}$ of crosslinking. Increased time at low $\mathrm{pH}$ can also result in a color change and increased brittleness of the TTM-CPnf- 2 membranes. The TTM-CPnf-2 membranes were partially water stable at a neutral $\mathrm{pH}$ for a short time interval (Table 3). Overall, the crosslinking step improved stability in water and retained nanofibrous morphologies in neutral and alkaline solutions after only a short exposure to GA. In acidic solutions, only those samples crosslinked for $3 \mathrm{~h}$ and above were insoluble. Alkaline solutions gave the best conditions to produce stable membrane mats.

\subsection{Swelling behavior of the composite nanofiber membrane}

The swelling behavior of TM-CPnf-2G was analyzed by immersing in $\mathrm{pH} 7$ (neutral), $\mathrm{pH} 10.01$ (alkaline), and $\mathrm{pH} 4.01$ (acidic) systems (Table 4). The swollen weights of CS/PEO after immersion in the different solutions were recorded for the immersion periods of 12 and $24 \mathrm{~h}$ time intervals. The highest swelling rate was determined to be $85.72 \%$ for acidic solutions and the lowest was recorded as $69.23 \%$ in basic solutions. The use of spinning solvents and crosslinking of CS/PEO may have enhanced the anti-water property of the blend [57] and moderated the water regain ability because a typical CS/PEO fiber can attain a maximal swelling ratio of $190 \%$ [58].

The experiment at $48 \mathrm{~h}$ was to investigate if the sorption equilibrium was attained after the initial $12 \mathrm{~h}$ immersion time. These results indicate that for $\mathrm{CS} / \mathrm{PEO}$ nanofiber membranes, the obtained 
Table 5

The lethal concentration values of TTM-CPnf-2 and TTM-CPnf-2G showing the lower and upper 95\% confidence limits (CL) for D. magna neonates.

\begin{tabular}{|c|c|c|c|c|c|}
\hline Nanofiber & Hour & Average LC20 (95\%CL) (mg/L) & Average LC50 (95\%CL) (mg/L) & $\mathrm{LOEC}(\mathrm{mg} / \mathrm{L})$ & $\operatorname{NOEC}(\mathrm{mg} / \mathrm{L})$ \\
\hline \multirow[t]{2}{*}{ TTM-CPnf-2 } & 24 & nd & nd & $>100$ & $\geq 100$ \\
\hline & 48 & nd & nd & $>100$ & $\geq 100$ \\
\hline \multirow[t]{2}{*}{ TTM-CPnf-2G } & 24 & 18.7 & 53.6 & 100 & 50 \\
\hline & 48 & 9.83 & 19.4 & 6.25 & 6.25 \\
\hline
\end{tabular}

NOEC, no observed effect concentration; nd, not detected; LOEC, low observed effect concentration.

values are almost constant in the $\mathrm{pH} 10.01$ (alkaline) and $\mathrm{pH} 7$ (neutral) solutions at different times, indicating the establishment of the equilibrium values. However, the highest swelling ratio was achieved under acidic $\mathrm{pH}$ (4.01) conditions for the CS/PEO but the water sorption in solutions after $24 \mathrm{~h}$ showed that the swelling ratio of the composite nanofiber membranes decreased. This observed weight loss could be because CS with very high DDA may be dispersible in a slightly acidic medium $(\mathrm{pH}<6)$ or due to some small quantity of the CS/PEO becoming soluble [59].

\subsection{TTM-CPnf-2G toxicity to Daphnia magna}

A disadvantage of GA in its unreacted form is that it is cytotoxic and has led to some reservations about its use in some applications [20]. CS is generally considered as non-toxic to animals [60] but after dissolution in acetic acid and after post-treatment by GA in the vapor phase, the product may induce acute or chronic toxicity [57]. Understanding biocompatibility is therefore crucial for using these nanomaterials in a way that is environmentally and biologically benign all through the product lifecycle and for use in other applications like the biomedical fields. The toxicities of TTM-CPnf-2 and TTM-CPnf-2G were tested and evaluated using the D. magna OECD 202 guidelines and the outcomes are shown in Table 5. Although research is carried out using $D$. magna models for toxicity evaluation, no studies have investigated the titanium-coated tracketched nanofiber membrane (TTM-CPnf) toxicity using this assessment model.

The LC 50 values of TTM-CPnf-2G are $53.6 \mathrm{mg} / \mathrm{L}$ and $19.4 \mathrm{mg} / \mathrm{L}$ at $24 \mathrm{~h}$ and $48 \mathrm{~h}$ respectively. The corresponding LC20 values were $18.7 \mathrm{mg} / \mathrm{L}$ and $9.83 \mathrm{mg} / \mathrm{L}$. Toxicity to daphnids was not observed with TTM-CPnf-2 due to low effect levels; the EC50 values not determined were considered as $100 \%$ for statistical analysis [61] and a lower EC50 value was indicative of higher toxicity to the tested organism. The outcome of the probit analysis with immobility at $24 \mathrm{~h}$, selected effective concentrations (LCX) of the test item, and the 95\%-confidence limits according to Fieller's theorem demonstrated that TTM-CPnf-2 will not trigger acute toxicity in D. magna. However, after 24 and 48 h exposure time using different concentrations of TTM-CPnf-2G solution, there was increased mortality that was concentration-dependent (Fig. 10) compared to the control (no TTM-CPnf composite). The concentration effect revealed that the TTM-CPnf-2G was hazardous to $D$. magna at higher concentrations ( 100 and $50 \mathrm{mg} / \mathrm{L}$ ) where $100 \%$ mortality of $D$. magna neonates was observed at $48 \mathrm{~h}$. This toxic effect at the highest concentration could be explained as either due to the release of strands of nanofiber, or an antibiotic effect induced by the $\mathrm{CS}$, or the release of Ti from the TM coating.

There was no mortality of D. magna in the control and in the lower concentrations of TTM-CPnf-2G samples after the exposure. Substances or materials affecting Daphnia at a $48 \mathrm{~h} \mathrm{LC/EC50} \mathrm{of} \mathrm{less}$ than $1 \mathrm{mg} / \mathrm{L}$ are classified as 'category acute 1 ' to aquatic organisms based on the globally harmonized system (GHS) of labeling and classification of chemicals [62]. However, European Union legislation and Regulation (EC) No. 1272/2008-classification, labeling, and packaging of substances and mixtures (CLP) [63] classified it otherwise as very toxic. Thus, based on these present results, the tested TTM-CPnf-2 and TTM-CPnf-2G cannot be classified to the 'category acute 1 ' based on the probit analysis.

TTM-CPnf-2 and TTM-CPnf-2G investigated samples were less toxic when compared to results obtained by Lv et al.[64] where the toxicological effects of carbon nanomaterials (graphene oxide) on D. magna were evaluated and the results of 72-h EC50 of $44.3 \mathrm{mg} / \mathrm{L}$ revealed non-severe acute toxicities of GO on D. magna. Zhu et al. [65] investigated the acute toxicity ( $48 \mathrm{~h}$ ) of water suspensions of six nanomaterials ( $\mathrm{nm}$ ) using D. magna and observed that the EC50 values of $0.622 \mathrm{mg} / \mathrm{L}$ for $\mathrm{ZnO}-\mathrm{nm}$ were the most toxic while

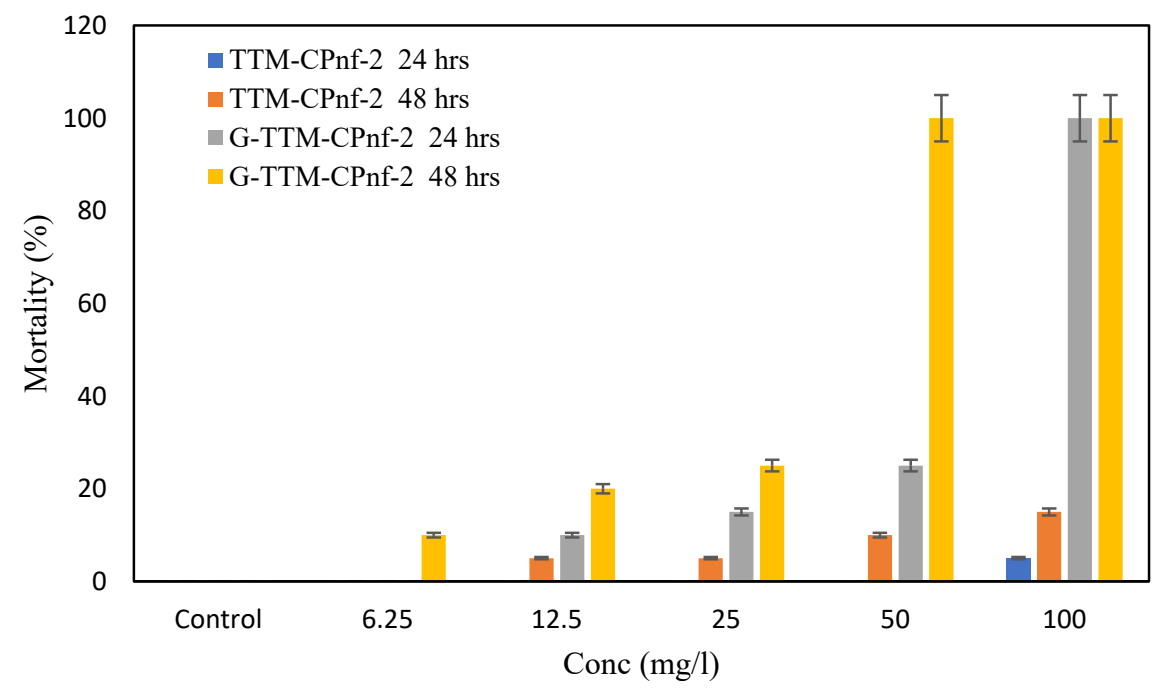

Fig. 10. Mortality of D. magna in different solution concentrations of TTM-CPnf-2 and TTM-CPnf-2G. 
$114.357 \mathrm{mg} / \mathrm{L}$ for $\mathrm{Al}_{2} \mathrm{O}_{3}-\mathrm{nm}$ had the least toxicity but nanomaterials from the test solutions were ingested by $D$. magna through feeding activities. Hyperbranched polymeric poly-amidoamine nanomaterials (Helux-3316) toxicity to D. magna was reported to be $16.8 \pm 1 \mathrm{mg} / \mathrm{L}$ and $4.69 \mathrm{mg} / \mathrm{L}$ for $24 \mathrm{~h}$ and $48 \mathrm{~h}$ EC50 values respectively [66] and was considered toxic because of the primary amines present in the chemical structure. The immobilization of D. magna on hyperbranched poly(ethyleneimine) was estimated to have $48 \mathrm{~h}$ EC50 values of $0.16 \mathrm{mg} / \mathrm{L}$ [67] but the toxicity of the $10 \mathrm{kDa}$ polymer increased with an increase in molecular weight. Composite CS/PEO nanofibers crosslinked with GA were deposited on titanium alloy disks and were found to bring significant improvements to the surface properties inducing durability and stronger antibacterial resistance [68]. Nevertheless, in the overall assessment of nanofiber toxicity, the material solubility, surface area, surface charge, exposure time, and TM coating are also anticipated to play vital roles, which will be investigated in future studies. The current study results showed that PEO blend and GA crosslinking diminished toxicity and thus improved the biocompatibility.

\section{Conclusion}

This study investigated composite membranes based on CS and PEO blend fabricated by electrospinning nanofibers on a Ti metallized TM as the membrane support and conductive cathode. The metallized TM membrane can be used as a collector in the process of electrospinning. The CS was blended with different amounts of PEO and electrospun effectively into nanofibers using acetic acid as the solvent at a CS/PEO polymer ratio of 70/30. GA-crosslinked CS/PEO $70 / 30$ (TTM-CPnf-2G) maintained the nanofiber morphologies in an aqueous environment and therefore increased the composite membrane's potential to be used as filters or adsorbents in acidic, neutral, or basic aqueous environments. The toxicity effects of TTMCPnf-2G indicated that it was not acutely toxic to D. magna testing conditions. The tailoring of CS:PEO blending and GA crosslinking was aimed at making the polymers more useful due to their biodegradability, which led to solubility control and impacted on toxicity. These results are significant because the titanium-coated track-etched nanofiber membrane composite extended the potential use of CS-based electrospun nanofiber in applications such as adsorption, separation, and filtration processes and in drug delivery platforms. This study provided novel insights and understanding into the biocompatibility potential of CS and PEO biodegradable nanomaterials and contributed to the understanding of the risk the composite nanomaterials may have on human health.

\section{Credit author statement}

O. Pereao: Methodology, Investigation, Writing-Original Draft. C. Uche: Investigation. P.S. Bublikov: Investigation, WritingReview \& Editing. C. Bode-Aluko: Investigation. A. Rossouw: Investigation. I.I. Vinogradov: Software, Investigation. A.N. Nechaev: Conceptualization, Methodology, Resources, Supervision, Project administration, Funding acquisition. B. Opeolu: Software, Resources, Writing-Review \& Editing, Supervision. L. Petrik: Conceptualization, Resources, Writing-Review \& Editing, Supervision, Project administration, Funding acquisition

All authors read and approved the final manuscript.

\section{Declaration of competing interest}

The authors declare that they have no known competing financial interests or personal relationships that could have appeared to influence the work reported in this paper.

\section{Acknowledgments}

The authors are grateful for the support from the National Research Foundation (JINR181129398), the Flerov Laboratory, Joint Institute for Nuclear Research, Environmental and Nanosciences Group, the University of the Western Cape, South Africa, and the Cape Peninsula University of Technology Postgraduate Research Scholarship.

\section{References}

[1] A. Boretti, L. Rosa, Reassessing the projections of the world water development report, NPJ Clean Water 2 (2019), https://doi.org/10.1038/s41545-0190039-9.

[2] J.N. Edokpayi, J.O. Odiyo, O.S. Durowoju, Impact of wastewater on surface water quality in developing countries: a case study of South Africa, in: Water Qual., InTech, 2017, https://doi.org/10.5772/66561.

[3] O.K. Pereao, C. Bode-Aluko, G. Ndayambaje, O. Fatoba, L.F. Petrik, Electrospinning: polymer nanofibre adsorbent applications for metal ion removal, J. Polym. Environ. 25 (2017) 1175-1189, https://doi.org/10.1007/s10924-016-0896-y.

[4] O. Pereao, C. Bode-Aluko, K. Laatikainen, A. Nechaev, L. Petrik, Morphology, modification and characterisation of electrospun polymer nanofiber adsorbent material used in metal ion removal, J. Polym. Environ. 27 (2019) 1843-1860, https://doi.org/10.1007/s10924-019-01497-w.

[5] L. Al-Naamani, S. Dobretsov, J. Dutta, J.G. Burgess, Chitosan-zinc oxide nanocomposite coatings for the prevention of marine biofouling, Chemosphere 168 (2017) 408-417, https://doi.org/10.1016/J.CHEMOSPHERE.2016.10.033.

[6] R. Augustine, S.R.U. Rehman, R. Ahmed, A.A. Zahid, M. Sharifi, M. Falahati, A. Hasan, Electrospun chitosan membranes containing bioactive and therapeutic agents for enhanced wound healing, Int. J. Biol. Macromol. 156 (2020) 153-170, https://doi.org/10.1016/j.ijbiomac.2020.03.207.

[7] I.I. Cardenas Bates, É. Loranger, B. Chabot, Chitosan-PEO nanofiber mats for copper removal in aqueous solution using a new versatile electrospinning collector, SN Appl. Sci. 2 (2020) 1-14, https://doi.org/10.1007/s42452-02003342-5.

[8] P. Kianfar, A. Vitale, S. DalleVacche, R. Bongiovanni, Photo-crosslinking of chitosan/poly(ethylene oxide) electrospun nanofibers, Carbohydr. Polym. 217 (2019) 144-151, https://doi.org/10.1016/j.carbpol.2019.04.062.

[9] A. Ahmed, L. Xu, J. Yin, M. Wang, F. Khan, M. Ali, High-throughput fabrication of chitosan/poly(ethylene oxide) nanofibers by modified free surface electrospinning, Fibers Polym. 21 (2020) 1945-1955, https://doi.org/10.1007/ s12221-020-1109-9.

[10] A. Saatchi, A.R. Arani, A. Moghanian, M. Mozafari, Synthesis and characterization of electrospun cerium-doped bioactive glass/chitosan/polyethylene oxide composite scaffolds for tissue engineering applications, Ceram. Int. 47 (2020) 260-271, https://doi.org/10.1016/j.ceramint.2020.08.130.

[11] N. Amiri, S. Ajami, A. Shahroodi, N. Jannatabadi, S. AmiriDarban, B.S. FazlyBazzaz, E. Pishavar, F. Kalalinia, J. Movaffagh, Teicoplanin-loaded chitosan-PEO nanofibers for local antibiotic delivery and wound healing, Int. J. Biol. Macromol. 162 (2020) 645-656, https://doi.org/10.1016/ j.ijbiomac.2020.06.195.

[12] P.Y. Apel, Track-etching, in: Encycl. Membr. Sci. Technol., John Wiley \& Sons, Inc., Hoboken, NJ, USA, 2013, pp. 1-25, https://doi.org/10.1002/ 9781118522318.emst040.

[13] P. Apel, Track etching technique in membrane technology, in: Radiat. Meas., Pergamon, 2001, pp. 559-566, https://doi.org/10.1016/S1350-4487(01)00228-1.

[14] M.R. Berber, Current advances of polymer composites for water treatment and desalination, J. Chem. 2020 (2020), https://doi.org/10.1155/2020/7608423.

[15] C.A. Bode-Aluko, K. Laatikainen, O. Pereao, A. Nechaev, I. Kochnev, A. Rossouw, S. Dobretsov, C. Branger, A. Sarbu, L. Petrik, Fabrication and characterisation of novel nanofiltration polymeric membrane, Mater. Today Commun. (2019) 100580, https://doi.org/10.1016/j.mtcomm.2019.100580.

[16] R.S. Barhate, C.K. Loong, S. Ramakrishna, Preparation and characterization of nanofibrous filtering media, J. Membr. Sci. 283 (2006) 209-218.

[17] L. Li, Y. Li, C. Yang, Chemical filtration of $\mathrm{Cr}$ (VI) with electrospun chitosan nanofiber membranes, Carbohydr. Polym. 140 (2016) 299-307.

[18] A. Podgórski, A. Bałazy, L. Gradoń, Application of nanofibers to improve the filtration efficiency of the most penetrating aerosol particles in fibrous filters, Chem. Eng. Sci. 61 (2006) 6804-6815.

[19] J.D. Schiffman, C.L. Schauer, One-step electrospinning of cross-linked Chitosan fibers, Biomacromolecules 8 (2007) 2665-2667, https://doi.org/10.1021/ bm7006983.

[20] M.S. Austero, A.E. Donius, U.G.K. Wegst, C.L. Schauer, New crosslinkers for electrospun chitosan fibre mats. I. Chemical analysis, J. R. Soc. Interface 9 (2012) 2551-2562, https://doi.org/10.1098/rsif.2012.0241.

[21] L. Li, Y. Li, L. Cao, C. Yang, Enhanced chromium (VI) adsorption using nanosized chitosan fibers tailored by electrospinning, Carbohydr. Polym. 125 (2015) 206-213, https://doi.org/10.1016/j.carbpol.2015.02.037.

[22] N.S. Surgutskaia, A. Di Martino, J. Zednik, K. Ozaltin, L. Lovecká, E.D. Bergerová, D. Kimmer, J. Svoboda, V. Sedlarik, Efficient $\mathrm{Cu} 2+, \mathrm{Pb} 2+$ and $\mathrm{Ni} 2+$ ion removal from wastewater using electrospun DTPA-modified chitosan/polyethylene 
oxide nanofibers, Separ. Purif. Technol. 247 (2020) 116914, https://doi.org/ 10.1016/j.seppur.2020.116914.

[23] S. Lopes, F. Ribeiro, J. Wojnarowicz, W. Lojkowski, K. Jurkschat, A. Crossley, A.M.V.M. Soares, S. Loureiro, Zinc oxide nanoparticles toxicity to Daphnia magna: size-dependent effects and dissolution, Environ. Toxicol. Chem. 33 (2014) 190-198, https://doi.org/10.1002/etc.2413.

[24] A. Bownik, Daphnia swimming behaviour as a biomarker in toxicity assessment: a review, Sci. Total Environ. 601-602 (2017) 194-205, https://doi.org/ 10.1016/j.scitotenv.2017.05.199.

[25] P.Y. Apel, S.N. Dmitriev, Micro- and nanoporous materials produced using accelerated heavy ion beams, Adv. Nat. Sci. Nanosci. Nanotechnol. 2 (2011), https://doi.org/10.1088/2043-6262/2/1/013002, 013002.

[26] O.V. Artoshina, F.O. Milovich, A. Rossouw, B.L. Gorberg, L.D. Iskhakova, R.P. Ermakov, V.K. Semina, Y.K. Kochnev, A.N. Nechaev, P.Y. Apel, Structure and phase composition of thin TiO2 films grown on the surface of metallized track-etched polyethylene terephthalate membranes by reactive magnetron sputtering, Inorg. Mater. 52 (2016) 945-954, https://doi.org/10.1134/ S0020168516080021.

[27] A. Rossouw, O.V. Artoshina, A.N. Nechaev, P.Y. Apel, L. Petrik, W.J. Perold, C.A. Pineda-Vargas, Stable ion beam analysis (RBS and PIXE) study of photocatalytictrack-etched membranes, in: Exot. Nucl., World Scientific, 2015, pp. 591-596, https://doi.org/10.1142/9789814632041_0065.

[28] X. Zhang, L. Ji, O. Toprakci, Y. Liang, M. Alcoutlabi, Electrospun nanofiber-based anodes, cathodes, and separators for advanced lithium-ion batteries, Polym. Rev. 51 (2011) 239-264, https://doi.org/10.1080/15583724.2011.593390.

[29] M.A. Kiechel, C.L. Schauer, Non-covalent crosslinkers for electrospun chitosan fibers, Carbohydr. Polym. 95 (2013) 123-133, https://doi.org/10.1016/ j.carbpol.2013.02.034.

[30] OECD, Test No. 202, Daphnia Sp. Acute Immobilisation Test, OECD, 2004, https://doi.org/10.1787/9789264069947-en.

[31] M. Heinlaan, M. Muna, K. Juganson, O. Oriekhova, S. Stoll, A. Kahru, V.I. Slaveykova, Exposure to sublethal concentrations of $\mathrm{Co3O} 4$ and $\mathrm{Mn} 2 \mathrm{O} 3$ nanoparticles induced elevated metal body burden in Daphnia magna, Aquat. Toxicol. 189 (2017) 123-133, https://doi.org/10.1016/j.aquatox.2017.06.002.

[32] B. Klüttgen, U. Dülmer, M. Engels, H.T. Ratte, ADaM, an artificial freshwater for the culture of zooplankton, Water Res. 28 (1994) 743-746, https://doi.org/ 10.1016/0043-1354(94)90157-0.

[33] B. Duan, C. Dong, X. Yuan, K. Yao, Electrospinning of chitosan solutions in acetic acid with poly (ethylene oxide), J. Biomater. Sci. Polym. Ed. 15 (2004) 797-811.

[34] D. Surendhiran, C. Li, H. Cui, L. Lin, Fabrication of high stability active nanofibers encapsulated with pomegranate peel extract using chitosan/PEO for meat preservation, Food Packag. Shelf Life 23 (2020) 100439, https://doi.org/ 10.1016/j.fpsl.2019.100439.

[35] L. Li, Y.-L. Hsieh, Chitosan bicomponentnanofibers and nanoporous fibers, Carbohydr. Res. 341 (2006) 374-381.

[36] Y.Z. Zhang, B. Su, S. Ramakrishna, C.T. Lim, Chitosan nanofibers from an easily electrospinnable UHMWPEO-doped chitosan solution system, Biomacromolecules 9 (2008) 136-141, https://doi.org/10.1021/bm701130e.

[37] S.I. Jeong, M.D. Krebs, C.A. Bonino, J.E. Samorezov, S.A. Khan, E. Alsberg, Electrospun chitosan-alginate nanofibers with in situ polyelectrolyte complexation for use as tissue engineering scaffolds, Tissue Eng. Part A 17 (2011) 59-70, https://doi.org/10.1089/ten.tea.2010.0086.

[38] M.B. Stie, M. Jones, H.O. Sørensen, J. Jacobsen, I.S. Chronakis, H.M. Nielsen, Acids 'generally recognized as safe' affect morphology and biocompatibility of electrospun chitosan/polyethylene oxide nanofibers, Carbohydr, Polym 215 (2019) 253-262, https://doi.org/10.1016/j.carbpol.2019.03.061.

[39] L. Martinová, D. Lubasová, Electrospun chitosan based nanofibers, Res. J. Text. Appar. 12 (2008) 72-79.

[40] X. Geng, O.H. Kwon, J. Jang, Electrospinning of chitosan dissolved in concentrated acetic acid solution, Biomaterials 26 (2005) 5427-5432, https://doi.org/ 10.1016/j.biomaterials.2005.01.066.

[41] O.K. Pereao, Functionalisation of ElectrospunNanofibre for Lanthanide Ion Adsorption from Aqueous Solution, University of the Western Cape, 2018. http://etd.uwc.ac.za/.

[42] Y.P. Singh, S. Dasgupta, S. Nayar, R. Bhaskar, Optimization of electrospinning process \& parameters for producing defect-free chitosan/polyethylene oxide nanofibers for bone tissue engineering, J. Biomater. Sci. Polym. Ed. 31 (2020) 781-803, https://doi.org/10.1080/09205063.2020.1718824.

[43] M. Spasova, N. Manolova, D. Paneva, I. Rashkov, Preparation of chitosancontaining nanofibres by electrospinning of chitosan/poly (ethylene oxide) blend solutions, E-Polymers 4 (2004).

[44] N. Bhattarai, D. Edmondson, O. Veiseh, F.A. Matsen, M. Zhang, Electrospun chitosan-based nanofibers and their cellular compatibility, Biomaterials 26 (2005) 6176-6184.

[45] M. Ignatova, K. Starbova, N. Markova, N. Manolova, I. Rashkov, Electrospunnano-fibre mats with antibacterial properties from quaternised chitosan and poly (vinyl alcohol), Carbohydr. Res. 341 (2006) 2098-2107.

[46] M. Pakravan, M.-C. Heuzey, A. Ajji, A fundamental study of chitosan/PEO electrospinning, Polymer 52 (2011) 4813-4824 (Guildf).

[47] C. Kriegel, K.M. Kit, D.J. McClements, J. Weiss, Electrospinning of chitosan-poly (ethylene oxide) blend nanofibers in the presence of micellar surfactant solutions, Polymer 50 (2009) 189-200 (Guildf).
[48] M.R. Kasaai, A review of several reported procedures to determine the degree of $\mathrm{N}$-acetylation for chitin and chitosan using infrared spectroscopy, Carbohydr. Polym. 71 (2008) 497-508.

[49] A. Zahir, Z. Aslam, M.S. Kamal, W. Ahmad, A. Abbas, R.A. Shawabkeh, Development of novel cross-linked chitosan for the removal of anionic Congo red dye, J. Mol. Liq. 244 (2017) 211-218, https://doi.org/10.1016/ j.molliq.2017.09.006.

[50] Y. Zhou, P. Qi, Z. Zhao, Q. Liu, Z. Li, Fabrication and characterization of fibrous HAP/PVP/PEO composites prepared by sol-electrospinning, RSC Adv. 4 (2014) 16731-16738, https://doi.org/10.1039/c3ra47168c.

[51] A.F.C. Rengifo, N.M. Stefanes, J. Toigo, C. Mendes, D.F. Argenta, M.E.R. Dotto, M.C. Santos da Silva, R.J. Nunes, T. Caon, A.L. Parize, E. Minatti, PEO-chitosan nanofibers containing carboxymethyl-hexanoyl chitosan/dodecyl sulfate nanoparticles loaded with pyrazoline for skin cancer treatment, Eur. Polym. J. 119 (2019) 335-343, https://doi.org/10.1016/j.eurpolymj.2019.08.001.

[52] S. Abid, T. Hussain, A. Nazir, A. Zahir, S. Ramakrishna, M. Hameed, N. Khenoussi, Enhanced antibacterial activity of PEO-chitosan nanofibers with potential application in burn infection management, Int. J. Biol. Macromol. 135 (2019) 1222-1236, https://doi.org/10.1016/j.ijbiomac.2019.06.022.

[53] I.E. Moreno-Cortez, J. Romero-García, V. González-González, D.I. GarcíaGutierrez, M.A. Garza-Navarro, R. Cruz-Silva, Encapsulation and immobilization of papain in electrospunnanofibrous membranes of PVA cross-linked with glutaraldehyde vapor, Mater. Sci. Eng. C 52 (2015) 306-314, https:// doi.org/10.1016/j.msec.2015.03.049.

[54] K. Govindasamy, N.A. Dahlan, P. Janarthanan, K.L. Goh, S.P. Chai, P. Pasbakhsh, Electrospun chitosan/polyethylene-oxide (PEO)/halloysites (HAL) membranes for bone regeneration applications, Appl. Clay Sci. 190 (2020) 105601, https:// doi.org/10.1016/j.clay.2020.105601.

[55] C.G.T. Neto, T.N.C. Dantas, J.L.C. Fonseca, M.R. Pereira, Permeability studies in chitosan membranes. Effects of crosslinking and poly(ethylene oxide) addition, Carbohydr. Res. 340 (2005) 2630-2636, https://doi.org/10.1016/ j.carres.2005.09.011.

[56] J.H. Park, Y.W. Cho, H. Chung, I.C. Kwon, S.Y. Jeong, Synthesis and characterization of sugar-bearing chitosan derivatives: aqueous solubility and biodegradability, Biomacromolecules 4 (2003) 1087-1091, https://doi.org/10.1021/ bm034094r.

[57] C.K. Chen, M.G. Liao, Y.L. Wu, Z.Y. Fang, J.A. Chen, Preparation of highly swelling/antibacterial cross-linked N-Maleoyl-Functional chitosan/polyethylene oxide nanofibermeshes for controlled antibiotic release, Mol. Pharm. $17 \quad$ (2020) 3461-3476, htps://doi.org/10.1021/ acs.molpharmaceut.0c00504.

[58] I. Kohsari, Z. Shariatinia, S.M. Pourmortazavi, Antibacterial electrospun chitosan-polyethylene oxide nanocomposite mats containing bioactive silver nanoparticles, Carbohydr. Polym. 140 (2016) 287-298, https://doi.org/ 10.1016/j.carbpol.2015.12.075.

[59] M.B. Stie, J.R. Gätke, F. Wan, I.S. Chronakis, J. Jacobsen, H.M. Nielsen, Swelling of mucoadhesiveelectrospun chitosan/polyethylene oxide nanofibers facilitates adhesion to the sublingual mucosa, Carbohydr. Polym. 242 (2020) 116428, https://doi.org/10.1016/j.carbpol.2020.116428.

[60] M. Friedman, V.K. Juneja, Review of antimicrobial and antioxidativeactivities of chitosans in food, J. Food Protect. 73 (2010) 1737-1761, https://doi.org/ 10.4315/0362-028X-73.9.1737.

[61] E. Mendonça, A. Picado, S.M. Paixão, L. Silva, M.A. Cunha, S. Leitão, I. Moura, C. Cortez, F. Brito, Ecotoxicity tests in the environmental analysis of wastewater treatment plants: case study in Portugal, J. Hazard Mater. 163 (2009) 665-670, https://doi.org/10.1016/j.jhazmat.2008.07.012.

[62] United Nations, Globally Harmonized System of Classification and Labelling of Chemicals (GHS), UN, 2019, https://doi.org/10.18356/f8fbb7cb-en.

[63] European Union, Regulation (EC) No 1272/2008 - Classification, Labelling and Packaging of Substances and Mixtures (CLP) - Safety and Health at Work - EUOSHA, 2015.

[64] X. Lv, Y. Yang, Y. Tao, Y. Jiang, B. Chen, X. Zhu, Z. Cai, B. Li, A mechanism study on toxicity of graphene oxide to Daphnia magna: direct link between bioaccumulation and oxidative stress, Environ. Pollut. 234 (2018) 953-959, https://doi.org/10.1016/j.envpol.2017.12.034.

[65] X. Zhu, L. Zhu, Y. Chen, S. Tian, Acute toxicities of six manufactured nanomaterial suspensions to Daphnia magna, J. Nanoparticle Res. 11 (2009) 67-75, https://doi.org/10.1007/s11051-008-9426-8.

[66] I. Martín-de-Lucía, F. Leganés, F. Fernández-Piñas, R. Rosal, Hyperbranched polymeric nanomaterials impair the freshwater crustacean Daphnia magna, Environ. Pollut. 249 (2019) 581-588, https://doi.org/10.1016/ j.envpol.2019.03.078.

[67] M. Salehi, R. Rodriguez, A. Boettcher, S. Powers, N. Geitner, D.A. Ladner, S. Rikard, A.J. Whelton, Impact of dispersant on early life stages of the water flea Daphnia magna and the eastern oyster Crassostreavirginica, J. Appl. Toxicol. 37 (2017) 1464-1470, https://doi.org/10.1002/jat.3494.

[68] F. Boschetto, H. Ngoc Doan, P. Phong Vo, M. Zanocco, W. Zhu, W. Sakai, T. Adachi, E. Ohgitani, N. Tsutsumi, O. Mazda, K. Kinashi, E. Marin, G. Pezzotti, Antibacterial and osteoconductiveeffects of chitosan/polyethylene oxide (PEO)/Bioactive glass nanofibers for orthopedic applications, Appl. Sci. 10 (2020) 2360, https://doi.org/10.3390/app10072360. 